\title{
Exploring triplet-quadruplet fermionic dark matter at the LHC and future colliders
}

\author{
Jin-Wei Wang, ${ }^{1,2, *}$ Xiao-Jun Bi, ${ }^{1, \dagger}$ Qian-Fei Xiang, ${ }^{1,2, \downarrow}$ Peng-Fei Yin, ${ }^{1, \S}$ and Zhao-Huan $\mathrm{Yu}^{3,4, \|}$ \\ ${ }^{1}$ Key Laboratory of Particle Astrophysics, Institute of High Energy Physics, Chinese Academy of Sciences, \\ Beijing 100049, China \\ ${ }^{2}$ School of Physical Sciences, University of Chinese Academy of Sciences, Beijing 100049, China \\ ${ }^{3}$ ARC Centre of Excellence for Particle Physics at the Terascale, School of Physics, \\ The University of Melbourne, Victoria 3010, Australia \\ ${ }^{4}$ School of Physics, Sun Yat-Sen University, Guangzhou 510275, China
}

(Received 20 November 2017; published 28 February 2018)

\begin{abstract}
We study the signatures of the triplet-quadruplet dark matter model at the LHC and future colliders, including the $100 \mathrm{TeV}$ Super Proton-Proton Collider and the $240 \mathrm{GeV}$ Circular Electron Positron Collider. The dark sector in this model contains one fermionic electroweak triplet and two fermionic quadruplets, which have two kinds of Yukawa couplings to the Higgs doublet. Electroweak production signals of the dark sector fermions in the monojet $+E_{\mathrm{T}}^{\dagger}$, disappearing track, and multilepton $+\mathscr{E}_{\mathrm{T}}$ channels at the LHC and the Super Proton-Proton Collider are investigated. Moreover, we study the loop effects of this model on the Circular Electron Positron Collider precision measurements of $e^{+} e^{-} \rightarrow Z h$ and $h \rightarrow \gamma \gamma$. We find that most of the parameter regions allowed by the observed dark matter relic density will be well explored by such direct and indirect searches at future colliders.
\end{abstract}

DOI: 10.1103/PhysRevD.97.035021

\section{INTRODUCTION}

With the discovery of the Higgs boson at the Large Hadron Collider (LHC) [1,2], the last missing piece of the standard model (SM) has been found. However, solid astrophysical and cosmological observations reveal the existence of dark matter (DM), which opens a door for exploring new physics beyond the standard model (BSM). Among various DM candidates proposed, weakly interacting massive particles (WIMPs) are very compelling, because they can naturally explain the DM relic density with particle masses of $\mathcal{O}(\mathrm{GeV})-\mathcal{O}(\mathrm{TeV})$ [3-6].

Many new physics models motivated by deep theoretical problems, e.g., supersymmetry (SUSY) models [7], naturally provide viable WIMP candidates. Nonetheless, in spite of other motivations, WIMP models can be easily constructed by introducing a dark sector with electroweak (EW) $\mathrm{SU}(2)_{\mathrm{L}}$ multiplets, whose neutral components

\footnotetext{
*wangjinwei@ihep.ac.cn

tbixj@ihep.ac.cn

*xiangqf@ihep.ac.cn

§yinpf@ihep.ac.cn

zhao-huan.yu@unimelb.edu.au
}

Published by the American Physical Society under the terms of the Creative Commons Attribution 4.0 International license. Further distribution of this work must maintain attribution to the author(s) and the published article's title, journal citation, and DOI. Funded by SCOAP. provide a potential DM candidate. A dark sector containing one nontrivial $\mathrm{SU}(2)_{\mathrm{L}}$ multiplet is considered a minimal extension, leading to the so-called minimal dark matter models [8-14]. Furthermore, introducing more than one $\mathrm{SU}(2)_{\mathrm{L}}$ multiplet in the dark sector gives rise to a much richer phenomenology [15-36].

Among EW gauge eigenstates in SUSY models, bino is an $\mathrm{SU}(2)_{\mathrm{L}}$ singlet, Higgsinos belong to a doublet, winos form a triplet. Thus, a dark sector with singlet and doublet fermions [15-18,20,23,27-30,36], or with doublet and triplet fermions $[21,29,36,37]$, is analogous to the EW sector of SUSY models in special limits, which has been well studied in the literature for decades. A more complicated case with triplet and quadruplet fermions [26,29], however, cannot be regarded as a limit of the SUSY electroweak sector. Thus, we may expect that the phenomenology could be quite different.

In such a triplet-quadruplet dark matter (TQDM) model, the dark sector involves one Weyl triplet with $Y=0$ and two Weyl quadruplets with $Y= \pm 1 / 2$. After the electroweak symmetry breaking (EWSB), these multiplets mix with each other; the mass eigenstates include three neutral Majorana fermions $\chi_{1,2,3}^{0}$, three singly charged fermions $\chi_{1,2,3}^{ \pm}$, and one doubly charged fermion $\chi^{ \pm \pm}$. By imposing a discrete $Z_{2}$ symmetry, the lightest neutral fermion $\chi_{1}^{0}$ is stable, serving as a DM candidate. In this work, we investigate signatures of the TQDM model at the LHC and future colliders. 
Through their EW gauge interactions, these dark sector fermions can be directly produced in $p p$ collisions, leading to unique signatures at the LHC, as well as at future $\sim 100 \mathrm{TeV} p p$ colliders, such as the Super Proton-Proton Collider (SPPC) [38,39] and the Future Circular Collider with hadron collisions (FCC-hh) [40]. All dark sector fermions would sequentially decay into the DM candidate $\chi_{1}^{0}$, which escapes detection and leads to a significant amount of missing transverse energy $\left(E_{\mathrm{T}}\right)$. Therefore, the monojet + $E_{\mathrm{T}}$ channel [41-44], where the final states are a large $E_{\mathrm{T}}$ associated with $\geq 1$ hard jet from initial state radiation (ISR), should be very efficient for tagging such signal events.

Besides, if $\chi_{1}^{0}$ consists of the pure triplet component or the pure quadruplet components, the mass splitting between $\chi_{1}^{ \pm}$ and $\chi_{1}^{0}$ would be $\sim \alpha_{2} m_{W} \sin ^{2}\left(\theta_{\mathrm{W}} / 2\right)=167 \mathrm{MeV}$, only induced by loop effects $[8,9,26]$. Consequently, the charged fermion $\chi_{1}^{ \pm}$has a macroscopic lifetime, and can travel a short distance in the inner detector before decaying to $\chi_{1}^{0}$ and a very soft, unlikely detected $\pi^{ \pm}$meson. This causes a disappearing track signature that has been well studied at the LHC $[12,45-50]$. Furthermore, if the mass splittings between $\chi_{1}^{0}$ and other dark sector fermions are close to or larger than $m_{W}$ and $m_{Z}$, the multilepton $+E_{\mathrm{T}}$ final states [51,52] could be utilized to probe the TQDM model.

Several projects of high energy $e^{+} e^{-}$colliders have been proposed, including the Circular Electron Positron Collider (CEPC) [38,39], the International Linear Collider (ILC) [53], and the Future Circular Collider with $e^{+} e^{-}$collisions (FCC-ee) [54]. At these $e^{+} e^{-}$colliders, a lot of Higgs and EW measurements with unprecedentedly high precisions, providing excellent indirect approaches to BSM electroweak multiplets. The sensitivity to the TQDM model via precision measurements of EW oblique parameters has been studied in Ref. [29]. In this paper, we investigate the impact of the TQDM model on the Higgs physics at the CEPC, including the loop effects on the $e^{+} e^{-} \rightarrow Z h$ production $[36,55,56]$ and the $h \rightarrow \gamma \gamma$ decay.

This paper is outlined as follows. In Sec. II we give a brief description of the TQDM model and analyze its mass spectrum. In Sec. III we investigate current constraints from the monojet $+E_{\mathrm{T}}$, disappearing track, and multilepton + $E_{\mathrm{T}}$ channels at the $13 \mathrm{TeV} \mathrm{LHC}$, and further study the prospects at the $100 \mathrm{TeV}$ SPPC based on Monte Carlo simulation. In Sec. IV, we calculate the one-loop corrections to the $e^{+} e^{-} \rightarrow Z h$ production cross section and $h \rightarrow \gamma \gamma$ partial width and estimate the CEPC prospects. Conclusions and further discussions are given in Sec. V.

\section{TRIPLET-QUADRUPLET DARK MATTER MODEL}

\section{A. Model Details}

In the TQDM model $[26,29]$, the dark sector involves one colorless left-handed Weyl triplet $T$ and two colorless left-handed Weyl quadruplets $Q_{1}$ and $Q_{2}$ obeying the following $S U(2)_{\mathrm{L}} \times U(1)_{\mathrm{Y}}$ gauge transformations:

$$
\begin{aligned}
T & =\left(\begin{array}{c}
T^{+} \\
T^{0} \\
-T^{-}
\end{array}\right) \in(\mathbf{3}, 0), \\
Q_{1} & =\left(\begin{array}{c}
Q_{1}^{+} \\
Q_{1}^{0} \\
Q_{1}^{-} \\
Q_{1}^{--}
\end{array}\right) \in\left(\mathbf{4},-\frac{1}{2}\right), \\
Q_{2} & =\left(\begin{array}{c}
Q_{2}^{++} \\
Q_{2}^{+} \\
Q_{2}^{0} \\
Q_{2}^{-}
\end{array}\right) \in\left(\mathbf{4}, \frac{1}{2}\right) .
\end{aligned}
$$

The hypercharge signs of the two quadruplets are opposite, which makes sure that the TQDM model is anomaly free. Gauge-invariant Lagrangians for the triplet and quadruplets are given by

$$
\mathcal{L}_{\mathrm{T}}=i T^{\dagger} \bar{\sigma}^{\mu} D_{\mu} T-\left(m_{T} a_{i j} T^{i} T^{j}+\text { H.c. }\right)
$$

and

$\mathcal{L}_{\mathrm{Q}}=i Q_{1}^{\dagger} \bar{\sigma}^{\mu} D_{\mu} Q_{1}+i Q_{2}^{\dagger} \bar{\sigma}^{\mu} D_{\mu} Q_{2}-\left(m_{Q} b_{i j} Q_{1}^{i} Q_{2}^{j}+\right.$ H.c. $)$,

where $D_{\mu}$ is the covariant derivative $D_{\mu} \equiv \partial_{\mu}-i g W_{\mu}^{a} \tau^{a}-$ $i g^{\prime} B_{\mu} Y$ with $\tau^{a} s$ being the generators for the corresponding $\mathrm{SU}(2)_{\mathrm{L}}$ representations. The constants $a_{i j}$ and $b_{i j}$ render the gauge invariance of the $a_{i j} T^{i} T^{j}$ and $b_{i j} Q_{1}^{i} Q_{2}^{j}$ terms, and can be decoded from Clebsch-Gordan (CG) coefficients multiplied by a factor to normalize the mass terms. The nonzero values are

$$
\begin{gathered}
a_{13}=a_{31}=\frac{1}{2}, \quad a_{22}=-\frac{1}{2} ; \\
b_{14}=b_{32}=1, \quad b_{23}=b_{41}=-1 .
\end{gathered}
$$

The Yukawa interactions between the dark sector multiplets and the Higgs doublet are given by

$$
\mathcal{L}_{\text {Yukawa }}=y_{1} c_{i j k} Q_{1}^{i} T^{j} H^{k}+y_{2} d_{i j k} Q_{2}^{i} T^{j} \tilde{H}^{k}+\text { H.c., }
$$

where $H$ is the Higgs doublet and $\tilde{H} \equiv i \sigma^{2} H^{*}$ expressed by 


$$
\begin{aligned}
& H=\left(\begin{array}{c}
H^{+} \\
H^{0}
\end{array}\right) \in\left(\mathbf{2}, \frac{1}{2}\right), \\
& \tilde{H}=i\left(\begin{array}{cc}
0 & -i \\
i & 0
\end{array}\right)\left(\begin{array}{c}
H^{-} \\
H^{0 *}
\end{array}\right)=\left(\begin{array}{c}
H^{0 *} \\
-H^{-}
\end{array}\right) \in\left(\mathbf{2},-\frac{1}{2}\right) .
\end{aligned}
$$

After the EWSB, the Higgs doublet obtains a vacuum expectation value $v$, and can be written in the unitary gauge as

$$
H=\frac{1}{\sqrt{2}}\left(\begin{array}{c}
0 \\
v+h
\end{array}\right)
$$

In the same way, by using the CG coefficients we can deduce the nonzero values of $c_{i j k}$ and $d_{i j k}$ are

$$
\begin{gathered}
c_{132}=-\frac{1}{\sqrt{2}}, \quad c_{222}=-\frac{1}{\sqrt{3}}, \quad c_{312}=\frac{1}{\sqrt{6}} ; \\
d_{231}=\frac{1}{\sqrt{6}}, \quad d_{321}=\frac{1}{\sqrt{3}}, \quad d_{411}=-\frac{1}{\sqrt{2}} .
\end{gathered}
$$

Thus, the explicit Yukawa interactions can be written as

$$
\begin{aligned}
& \mathcal{L}_{\text {Yukawa }} \\
& =y_{1}(v+h)\left(\frac{1}{\sqrt{6}} Q_{1}^{-} T^{+}-\frac{1}{\sqrt{3}} Q_{1}^{0} T^{0}-\frac{1}{\sqrt{2}} Q_{1}^{+} T^{-}\right) \\
& +y_{2}(v+h)\left(-\frac{1}{\sqrt{2}} Q_{2}^{-} T^{+}+\frac{1}{\sqrt{3}} Q_{2}^{0} T^{0}+\frac{1}{\sqrt{6}} Q_{2}^{+} T^{-}\right)+\text {H.c. }
\end{aligned}
$$

Then we rewrite all the mass terms into a matrix form:

$$
\begin{aligned}
\mathcal{L}_{\text {mass }}= & -\frac{1}{2}\left(\begin{array}{lll}
T^{0} & Q_{1}^{0} & Q_{2}^{0}
\end{array}\right) \mathcal{M}_{\mathrm{N}}\left(\begin{array}{c}
T^{0} \\
Q_{1}^{0} \\
Q_{2}^{0}
\end{array}\right) \\
& -\left(\begin{array}{lll}
T^{-} & Q_{1}^{-} & Q_{2}^{-}
\end{array}\right) \mathcal{M}_{\mathrm{C}}\left(\begin{array}{c}
T^{+} \\
Q_{1}^{+} \\
Q_{2}^{+}
\end{array}\right) \\
& -m_{\mathrm{Q}} Q_{1}^{--} Q_{2}^{++}+\text {H.c. } \\
= & -\frac{1}{2} \sum_{i=1}^{3} m_{\chi_{i}^{0}} \chi_{i}^{0} \chi_{i}^{0}-\sum_{i=1}^{3} m_{\chi_{i}^{ \pm} \chi_{i}^{-} \chi_{i}^{+}} \\
& -m_{\chi^{ \pm \pm} \chi^{--} \chi^{++}+\text {H.c. },}
\end{aligned}
$$

where $\chi_{i}^{0}, \chi_{i}^{ \pm}$, and $\chi^{ \pm \pm}$are mass eigenstates, and $m_{\chi_{i}^{0}}, m_{\chi_{i}^{ \pm}}$, and $m_{\chi^{ \pm \pm}}$are the masses of corresponding mass eigenstates. The mass matrixes $\mathcal{M}_{\mathrm{N}}$ and $\mathcal{M}_{\mathrm{C}}$ are given by

$$
\begin{aligned}
& \mathcal{M}_{\mathrm{N}}=\left(\begin{array}{ccc}
m_{T} & \frac{1}{\sqrt{3}} y_{1} v & -\frac{1}{\sqrt{3}} y_{2} v \\
\frac{1}{\sqrt{3}} y_{1} v & 0 & m_{Q} \\
-\frac{1}{\sqrt{3}} y_{2} v & m_{Q} & 0
\end{array}\right), \\
& \mathcal{M}_{\mathrm{C}}=\left(\begin{array}{ccc}
m_{T} & \frac{1}{\sqrt{2}} y_{1} v & -\frac{1}{\sqrt{6}} y_{2} v \\
-\frac{1}{\sqrt{6}} y_{1} v & 0 & -m_{Q} \\
\frac{1}{\sqrt{2}} y_{2} v & -m_{Q} & 0
\end{array}\right), \\
& m_{\chi^{ \pm \pm}}=m_{Q} .
\end{aligned}
$$

We can use three unitary matrixes $\mathcal{N}, \mathcal{C}_{\mathrm{L}}$, and $\mathcal{C}_{\mathrm{R}}$ to diagonalize $\mathcal{M}_{\mathrm{N}}$ and $\mathcal{M}_{\mathrm{C}}$ :

$$
\begin{aligned}
\mathcal{N}^{T} \mathcal{M}_{\mathrm{N}} \mathcal{N} & =\operatorname{diag}\left(m_{\chi_{1}^{0}}, m_{\chi_{2}^{0}}, m_{\chi_{3}^{0}}\right), \\
\mathcal{C}_{\mathrm{R}}^{T} \mathcal{M}_{\mathrm{C}} \mathcal{C}_{\mathrm{L}} & =\operatorname{diag}\left(m_{\chi_{1}^{ \pm}}, m_{\chi_{2}^{ \pm}}, m_{\chi_{3}^{ \pm}}\right) .
\end{aligned}
$$

Thus, the mass eigenstates are linked to the gauge eigenstates via

$$
\begin{aligned}
\left(\begin{array}{l}
T^{0} \\
Q_{1}^{0} \\
Q_{2}^{0}
\end{array}\right)=\mathcal{N}\left(\begin{array}{l}
\chi_{1}^{0} \\
\chi_{2}^{0} \\
\chi_{3}^{0}
\end{array}\right), \quad\left(\begin{array}{c}
T^{+} \\
Q_{1}^{+} \\
Q_{2}^{+}
\end{array}\right)=\mathcal{C}_{L}\left(\begin{array}{c}
\chi_{1}^{+} \\
\chi_{2}^{+} \\
\chi_{3}^{+}
\end{array}\right), \\
\left(\begin{array}{l}
T^{-} \\
Q_{1}^{-} \\
Q_{2}^{-}
\end{array}\right)=\mathcal{C}_{R}\left(\begin{array}{c}
\chi_{1}^{-} \\
\chi_{2}^{-} \\
\chi_{3}^{-}
\end{array}\right) .
\end{aligned}
$$

For convenience, we adopt the mass orders $0 \leq m_{\chi_{1}^{0}} \leq$ $m_{\chi_{2}^{0}} \leq m_{\chi_{3}^{0}}$ and $0 \leq m_{\chi_{1}^{ \pm}} \leq m_{\chi_{2}^{ \pm}} \leq m_{\chi_{3}^{ \pm}}$, which can be realized by adjusting the $\mathcal{N}, \mathcal{C}_{\mathrm{L}}$, and $\mathcal{C}_{\mathrm{R}}$. Because of the discrete $Z_{2}$ symmetry, the lightest Majorana fermion $\chi_{1}^{0}$ would be the DM candidate if it is the lightest dark sector fermion. Note that we will not consider any $C P$-violation phase in this work, and thus there are only four independent parameters in the TQDM model: $m_{T}, m_{Q}, y_{1}$, and $y_{2}$.

Besides, we can construct 4-component Dirac spinors from 2-component Weyl spinors:

$\Psi_{i}=\left(\begin{array}{c}\chi_{i}^{0} \\ \left(\chi_{i}^{0}\right)^{\dagger}\end{array}\right), \quad X_{i}^{-}=\left(\begin{array}{c}\chi_{i}^{-} \\ \left(\chi_{i}^{+}\right)^{\dagger}\end{array}\right), \quad X^{--}=\left(\begin{array}{c}\chi^{--} \\ \left(\chi^{++}\right)^{\dagger}\end{array}\right)$.

The mass and kinetic terms can be rewritten as

$$
\begin{aligned}
& \mathcal{L}_{\text {mass }}=-\frac{1}{2} m_{\chi_{i}^{0}} \overline{\Psi_{i}} \Psi_{i}-m_{\chi_{i}^{ \pm}} \overline{X_{i}^{-}} X_{i}^{-}-m_{\chi^{ \pm \pm}} \overline{X^{--}} X^{--} \\
& \mathcal{L}_{\text {kinetic }}=\frac{1}{2} i \overline{\Psi_{i}} \gamma^{\mu} \partial_{\mu} \Psi_{i}+i \overline{X_{i}^{-}} \gamma^{\mu} \partial_{\mu} X_{i}^{-}+i \overline{X^{--}} \gamma^{\mu} \partial_{\mu} X^{--} .
\end{aligned}
$$

The Yukawa interaction terms are 


$$
\begin{aligned}
\mathcal{L}_{\text {Yukawa }}= & \left(-\frac{y_{1}}{\sqrt{2}} \mathcal{C}_{\mathrm{L}, 2 i} \mathcal{C}_{\mathrm{R}, 1 j}+\frac{y_{1}}{\sqrt{6}} \mathcal{C}_{\mathrm{L}, 1 i} \mathcal{C}_{\mathrm{R}, 2 j}+\frac{y_{2}}{\sqrt{6}} \mathcal{C}_{\mathrm{L}, 3 i} \mathcal{C}_{\mathrm{R}, 1 j}-\frac{y_{2}}{\sqrt{2}} \mathcal{C}_{\mathrm{L}, 1 i} \mathcal{C}_{\mathrm{R}, 3 j}\right) \overline{X_{i}^{-}} P_{\mathrm{L}} X_{j}^{-} h \\
& +\left(-\frac{y_{1}}{\sqrt{2}} \mathcal{C}_{\mathrm{R}, 1 i}^{*} \mathcal{C}_{\mathrm{L}, 2 j}^{*}+\frac{y_{1}}{\sqrt{6}} \mathcal{C}_{\mathrm{R}, 2 i}^{*} \mathcal{C}_{\mathrm{L}, 1 j}^{*}+\frac{y_{2}}{\sqrt{6}} \mathcal{C}_{\mathrm{R}, 1 i}^{*} \mathcal{C}_{\mathrm{L}, 3 j}^{*}-\frac{y_{2}}{\sqrt{2}} \mathcal{C}_{\mathrm{R}, 3 i}^{*} \mathcal{C}_{\mathrm{L}, 1 j}^{*}\right) \overline{X_{i}^{-}} P_{\mathrm{R}} X_{j}^{-} h \\
& -\left(\frac{y_{1}}{\sqrt{3}} \mathcal{N}_{2 i} \mathcal{N}_{1 j}-\frac{y_{2}}{\sqrt{3}} \mathcal{N}_{3 i} \mathcal{N}_{1 j}\right) \overline{\Psi_{i}} P_{\mathrm{L}} \Psi_{j} h-\left(\frac{y_{1}}{\sqrt{3}} \mathcal{N}_{1 i}^{*} \mathcal{N}_{2 j}^{*}-\frac{y_{2}}{\sqrt{3}} \mathcal{N}_{1 i}^{*} \mathcal{N}_{3 j}^{*}\right) \overline{\Psi_{i}} P_{\mathrm{R}} \Psi_{j} h .
\end{aligned}
$$

The interaction terms with the photon are

$$
\mathcal{L}_{\text {photon }}=-e A_{\mu} \overline{X_{i}^{-}} \gamma^{\mu} X_{i}^{-}-2 e A_{\mu} \overline{X^{--}} \gamma^{\mu} X^{--}
$$

The interaction terms with the $Z$ boson are

$$
\begin{aligned}
\mathcal{L}_{\mathrm{Z}}= & \left(-c_{\mathrm{W}} g \mathcal{C}_{\mathrm{R}, 1 i}^{*} \mathcal{C}_{\mathrm{R}, 1 j}+\frac{g\left(s_{\mathrm{W}}^{2}-c_{\mathrm{W}}^{2}\right)}{2 c_{\mathrm{W}}} \mathcal{C}_{\mathrm{R}, 2 i}^{*} \mathcal{C}_{\mathrm{R}, 2 j}-\frac{g\left(3 c_{\mathrm{W}}^{2}+s_{\mathrm{W}}^{2}\right)}{2 c_{\mathrm{W}}} \mathcal{C}_{\mathrm{R}, 3 i}^{*} \mathcal{C}_{\mathrm{R}, 3 j}\right) \overline{X_{i}^{-}} \gamma^{\mu} P_{\mathrm{L}} X_{j}^{-} Z_{\mu} \\
& +\left(-c_{\mathrm{W}} g \mathcal{C}_{\mathrm{L}, 1 i} \mathcal{C}_{\mathrm{L}, 1 j}^{*}-\frac{g\left(3 c_{\mathrm{W}}^{2}+s_{\mathrm{W}}^{2}\right)}{2 c_{\mathrm{W}}} \mathcal{C}_{\mathrm{L}, 2 i} \mathcal{C}_{\mathrm{L}, 2 j}^{*}+\frac{g\left(s_{\mathrm{W}}^{2}-c_{\mathrm{W}}^{2}\right)}{2 c_{\mathrm{W}}} \mathcal{C}_{\mathrm{L}, 3 i} \mathcal{C}_{\mathrm{L}, 3 j}^{*}\right) \overline{X_{i}^{-}} \gamma^{\mu} P_{\mathrm{R}} X_{j}^{-} Z_{\mu} \\
& +\frac{g}{4 c_{\mathrm{W}}}\left(\mathcal{N}_{2 i}^{*} \mathcal{N}_{2 j}-\mathcal{N}_{3 i}^{*} \mathcal{N}_{3 j}\right) \overline{\Psi_{i}} \gamma^{\mu} P_{\mathrm{L}} \Psi_{j} Z_{\mu}-\frac{g}{4 c_{\mathrm{W}}}\left(\mathcal{N}_{2 i} \mathcal{N}_{2 j}^{*}-\mathcal{N}_{3 i} \mathcal{N}_{3 j}^{*}\right) \overline{\Psi_{i}} \gamma^{\mu} P_{\mathrm{R}} \Psi_{j} Z_{\mu}+\frac{g\left(s_{\mathrm{W}}^{2}-3 c_{\mathrm{W}}^{2}\right)}{2 c_{\mathrm{W}}} \overline{X^{--}} \gamma^{\mu} X^{--} Z_{\mu} .
\end{aligned}
$$

Here $s_{\mathrm{W}} \equiv \sin \theta_{\mathrm{W}}$ and $c_{\mathrm{W}} \equiv \cos \theta_{\mathrm{W}}$ with $\theta_{\mathrm{W}}$ denoting the Weinberg angle. Finally, the interaction terms with the $W$ boson are

$$
\begin{aligned}
\mathcal{L}_{\mathrm{W}}= & g\left(-\mathcal{N}_{1 i}^{*} \mathcal{C}_{\mathrm{R}, 1 j}+\sqrt{2} \mathcal{N}_{2 i}^{*} \mathcal{C}_{\mathrm{R}, 2 j}+\frac{\sqrt{6}}{2} \mathcal{N}_{3 i}^{*} \mathcal{C}_{\mathrm{R}, 3 j}\right) \bar{\Psi}_{i} \gamma^{\mu} P_{\mathrm{L}} X_{j}^{-} W_{\mu}^{+} \\
& -g\left(\mathcal{N}_{1 i} \mathcal{C}_{\mathrm{L}, 1 j}^{*}+\frac{\sqrt{6}}{2} \mathcal{N}_{2 i} \mathcal{C}_{\mathrm{L}, 2 j}^{*}+\sqrt{2} \mathcal{N}_{3 i} \mathcal{C}_{\mathrm{L}, 3 j}^{*}\right) \overline{\Psi_{i}} \gamma^{\mu} P_{\mathrm{R}} X_{j}^{-} W_{\mu}^{+} \\
& +g\left(-\mathcal{C}_{R, 1 i}^{*} \mathcal{N}_{1 j}+\sqrt{2} \mathcal{C}_{\mathrm{R}, 2 i}^{*} \mathcal{N}_{2 j}+\frac{\sqrt{6}}{2} \mathcal{C}_{\mathrm{R}, 3 i}^{*} \mathcal{N}_{3 j}\right) \overline{X_{i}^{-}} \gamma^{\mu} P_{\mathrm{L}} \Psi_{j} W_{\mu}^{-} \\
& -g\left(\mathcal{C}_{\mathrm{L}, 1 i} \mathcal{N}_{1 j}^{*}+\frac{\sqrt{6}}{2} \mathcal{C}_{\mathrm{L}, 2 i} \mathcal{N}_{2 j}^{*}+\sqrt{2} \mathcal{C}_{\mathrm{L}, 3 i} \mathcal{N}_{3 j}^{*}\right) \overline{X_{i}^{-}} \gamma^{\mu} P_{\mathrm{R}} \Psi_{j} W_{\mu}^{-} \\
& +\frac{\sqrt{6}}{2} g \mathcal{C}_{\mathrm{R}, 2 i}^{*} \overline{X_{i}^{-}} \gamma^{\mu} P_{\mathrm{L}} X^{--} W_{\mu}^{+}-\frac{\sqrt{6}}{2} g \mathcal{C}_{\mathrm{L}, 3 i} \overline{X_{i}^{-}} \gamma^{\mu} P_{\mathrm{R}} X^{--} W_{\mu}^{+} \\
& +\frac{\sqrt{6}}{2} g \mathcal{C}_{\mathrm{R}, 2 i} \overline{X^{--}} \gamma^{\mu} P_{\mathrm{L}} X_{i}^{-} W_{\mu}^{-}-\frac{\sqrt{6}}{2} g \mathcal{C}_{\mathrm{L}, 3 i}^{*} \overline{X^{--}} \gamma^{\mu} P_{\mathrm{R}} X_{i}^{-} W_{\mu}^{-}
\end{aligned}
$$

\section{B. Mass spectrum}

Masses of dark sector fermions and are determined by the parameter set $\left(m_{T}, m_{Q}, y_{1}, y_{2}\right)$. As the mass spectrum significantly affects the kinematics of their production and decay processes at colliders, we carry out a careful calculation for the masses with one-loop corrections. Details of the calculation are not described in this paper, but interested readers may refer to Refs. [26,57-59]. In some parameter regions with $y_{1} y_{2}<0$, the condition of $m_{\chi_{1}^{0}}<m_{\chi_{1}^{ \pm}}$satisfied at tree level may not hold at one-loop level [26]. Such parameter regions should be excluded, since there is no available DM candidate.

There are some symmetries regarding the Yukawa couplings. If one exchanges the values of $y_{1}$ and $y_{2}$ $\left(y_{1} \leftrightarrow y_{2}\right)$, or simultaneously change the signs of $y_{1}$ and $y_{2}\left(y_{1} \rightarrow-y_{1}, y_{2} \rightarrow-y_{2}\right)$, the mass spectrum would not change. Another interesting feature is that the dark sector respects a global custodial symmetry when $y_{1}= \pm y_{2}$ $[26,29]$. This custodial symmetry ensures that $m_{\chi_{i}^{0}}=$ $m_{\chi_{i}^{ \pm}}(i=1,2,3)$ at tree level. At one-loop level, the limit 


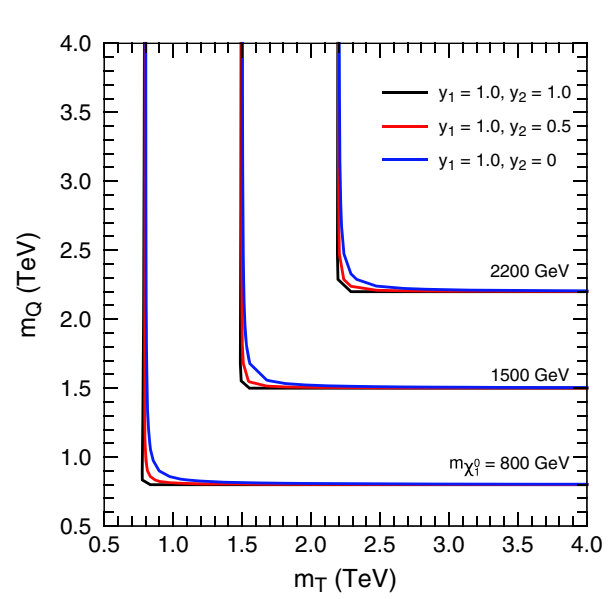

(a) $m_{\chi_{1}^{0}}$ contours

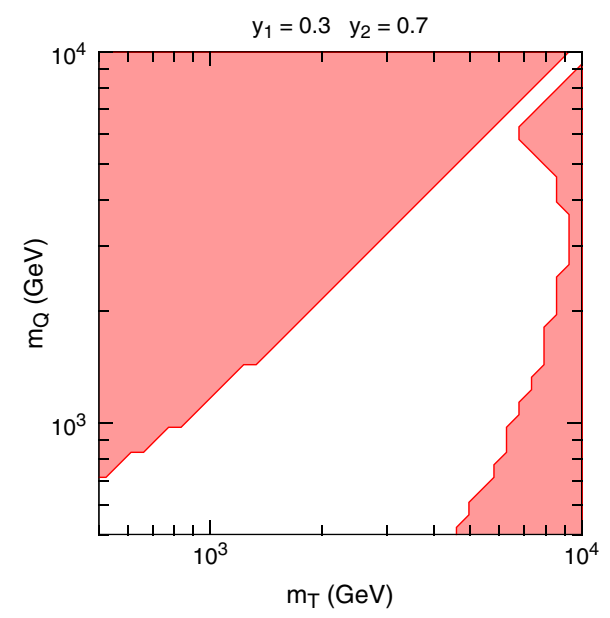

(c) $y_{1}=0.3, y_{2}=0.7$

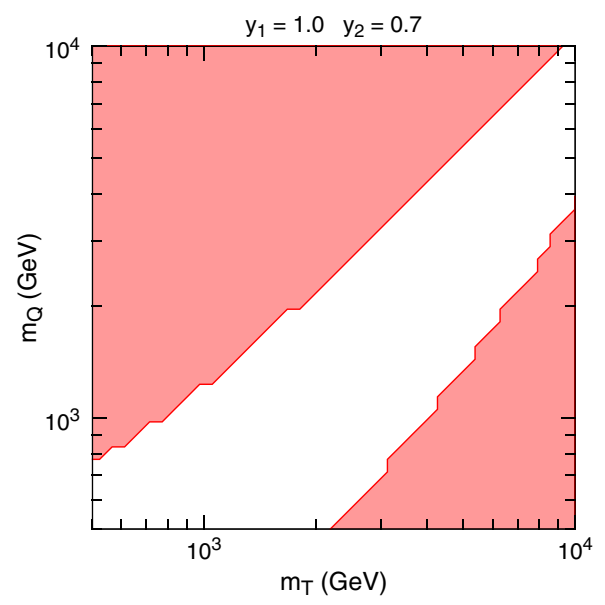

(b) $y_{1}=1.0, y_{2}=0.7$

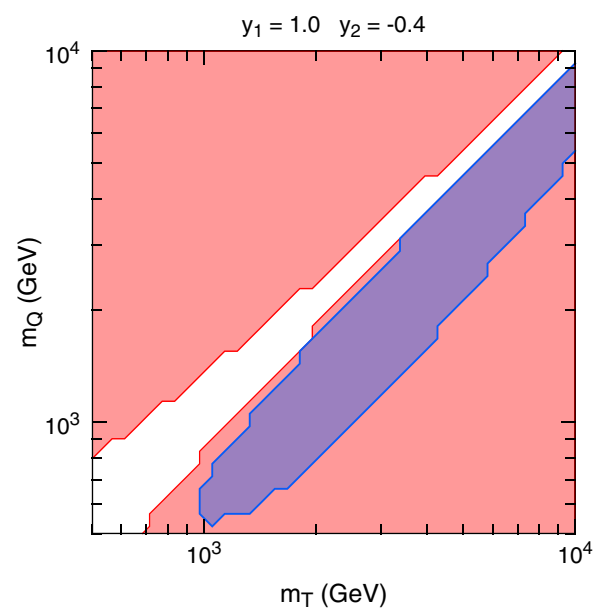

(d) $y_{1}=1.0, y_{2}=-0.4$

FIG. 1. The impacts of $y_{1}$ and $y_{2}$ values on $m_{\chi_{1}^{0}}$ (a) and the mass splitting $m_{\chi_{1}^{ \pm}}-m_{\chi_{1}^{0}}$ (b)-(d) in the $m_{T^{-}} m_{Q}$ plane. In the red regions, $m_{\chi_{1}^{ \pm}}-m_{\chi_{1}^{0}}$ is smaller than $250 \mathrm{MeV}$ at one-loop level. In the blue region, $m_{\chi_{1}^{ \pm}}-m_{\chi_{1}^{0}}$ is negative at one loop-level, and thus $\chi_{1}^{0}$ fails to be a DM candidate.

$y_{1}=-y_{2}$ leads to $m_{\chi_{1}^{0}}>m_{\chi_{1}^{ \pm}}$and hence is not interested. On the other hand, one-loop corrections in the limit $y_{1}=y_{2}$ lift the masses of charged fermions, resulting in $m_{\chi_{i}^{ \pm}}-m_{\chi_{i}^{0}} \simeq \mathcal{O}(100) \mathrm{MeV}$. Such a small mass splitting could give rise to a disappearing track signature at colliders.

In Fig. 1(a), we show the contours of $m_{\chi_{1}^{0}}$ for different sets of $y_{1}$ and $y_{2}$ in the $m_{T}-m_{Q}$ plane. We can see that when $m_{T}$ and $m_{Q}$ are $\gtrsim 1 \mathrm{TeV}, y_{1}$ and $y_{2}$ do not significantly affect the value of $m_{\chi_{1}^{0}}$. This is because the mixing mass terms between the triplet and quadruplets are just determined by the Yukawa interactions at the order of the EWSB scale $\sim \mathcal{O}(100) \mathrm{GeV}$. In this case, $m_{\chi_{1}^{0}}$ is close to either $m_{T}$ or $m_{Q}$, depending on which one is smaller. Consequently, collider searches for these parameter regions may not be sensitive to $y_{1}$ and $y_{2}$, and can set general limits on $m_{T}$ and $m_{Q}$.
Figures 1(b), 1(c), and 1(d) demonstrate special regions for the mass splitting $m_{\chi_{1}^{ \pm}}-m_{\chi_{1}^{0}}$ with three sets of Yukawa couplings. The red regions denote the regions where $m_{\chi_{1}^{ \pm}}-m_{\chi_{1}^{0}}$ is smaller than $250 \mathrm{MeV}$ at one-loop level. In such regions, the disappearing track channel could be quite sensitive. The blue region in Fig. 1(d) corresponds to $m_{\chi_{1}^{0}}>m_{\chi_{1}^{ \pm}}$, which excludes $\chi_{1}^{0}$ as a DM candidate. It can be seen that small mass splittings are quite common in this model, even for the case of $y_{1} \neq y_{2}$. Therefore, it is worthy of considering disappearing track searches at the LHC and future colliders.

\section{LHC AND SPPC SEARCHES}

In this section, we investigate the current LHC constraints on the TQDM model in several search channels by reinterpreting ATLAS analyses at $\sqrt{s}=13 \mathrm{TeV}$. We 
further explore the prospect of the future $p p$ collider SPPC, based on Monte Carlo simulation. The collision energy and the integrated luminosity of the SPPC are set to be $100 \mathrm{TeV}$ and $3 \mathrm{ab}^{-1}$, respectively.

In our simulation, FeynRules 2.3.26 [60] is employed to implement the TQDM model. Signal and background samples are generated by MadGraph 5.2.1.2 [61] at parton level. Pythia 6.4.28 [62] is used to deal with parton showering, hadronization, and decay processes. Background events are matched up to two additional jets with the MLM matching scheme [63], while signal events are matched with one jet for simplicity. We have checked that the difference between 1-jet matching and 2-jet matching for signals is negligible. Delphes 3.3.3 [64] is utilized to perform a fast detector simulation.

\section{A. Monojet $+E_{\mathrm{T}}$ channel}

First, we consider the monojet $+E_{\mathrm{T}}$ channel, where the final state involves an energetic jet and a large missing transverse momentum. This channel is clean and distinctive and has been widely used to search for large extra dimensions [65], SUSY models [66], and generic WIMPs $[41,42,44,67]$ at the Tevatron and the LHC. In the TQDM model, pair production of dark sector fermions associated with a hard ISR jet would also give rise to such a monojet $+\mathscr{E}_{\mathrm{T}}$ final state.

$\mathrm{SM}$ backgrounds in the monojet $+E_{\mathrm{T}}$ channel are dominated by $Z(\rightarrow \nu \bar{\nu})+$ jets, $W(\rightarrow \ell \nu)+$ jets, $t \bar{t}+$ jets, and $W(\rightarrow \ell \nu) W(\rightarrow \ell \nu)+$ jets $[44,45]$. Other contributions, e.g., from top production associated with additional vector bosons can be neglected [44]. We have carefully compared our simulated backgrounds with those given by the ATLAS monojet $+E_{\mathrm{T}}$ analysis with $\sqrt{s}=13 \mathrm{TeV}$ [43], and find that they are almost perfectly matched with each other. For the signal simulation, we include all the production processes of $p p \rightarrow \chi \chi+$ jets, where $\chi$ represents any fermion in $\left(\chi_{i}^{0}, \chi_{i}^{ \pm}, \chi^{ \pm \pm}\right)$.

For evaluating the current LHC constraint, we study the latest result from the ATLAS analysis [44] with $\sqrt{s}=13 \mathrm{TeV}$ and an integrated luminosity of $36.1 \mathrm{fb}^{-1}$. The object reconstruction conditions and kinematic cut conditions used in this analysis are listed in the second column of Table I. They require the final state involving at least one energetic central jet, a large $E_{\mathrm{T}}$, and no lepton $\left(n_{\ell}=0\right.$ for $\left.\ell=e, \mu\right)$. We closely follow these cut conditions to reinterpret the experimental result. The resulting constraint is shown in Fig. 2, where the orange regions are excluded at $95 \%$ C.L. by the LHC search.

If $m_{Q} \gg m_{T}, \chi_{1}^{0}$ is dominated by the triplet component, and the LHC bound can exclude up to $m_{\chi_{1}^{0}} \sim 70 \mathrm{GeV}$. On the other hand, $m_{T} \gg m_{Q}$ leads to a quadruplet-dominated $\chi_{1}^{0}$, and the LHC exclusion limit goes up to $m_{\chi_{1}^{0}} \sim 200 \mathrm{GeV}$. This is due to the fact that the pure quadruplets have larger production cross section than the pure triplet, as
TABLE I. Reconstruction and cut conditions in the monojet + $E_{\mathrm{T}}$ channel used in the ATLAS analysis at the $13 \mathrm{TeV}$ LHC [44] and those adopted for estimating the sensitivity at the $100 \mathrm{TeV}$ SPPC. Here $j$ and $\ell=e, \mu$ represent jet and lepton, respectively. $j_{1}$ means the leading jet, i.e., the jet with the largest $p_{\mathrm{T}} . n_{\ell}$ and $n_{j}$ are the numbers of reconstructed leptons and jets, respectively. $\not \not_{\mathrm{T}}$ denotes the missing transverse momentum vector.

\begin{tabular}{lcc}
\hline \hline & $13 \mathrm{TeV}$ LHC & $100 \mathrm{TeV}$ SPPC \\
\hline \multicolumn{3}{c}{ Reconstruction Conditions } \\
$p_{\mathrm{T}}(j),|\eta(j)|$ & $>30 \mathrm{GeV},<2.8$ & $>80 \mathrm{GeV},<2.8$ \\
$p_{\mathrm{T}}(e),|\eta(e)|$ & $>20 \mathrm{GeV},<2.47$ & $>20 \mathrm{GeV},<2.47$ \\
$p_{\mathrm{T}}(\mu),|\eta(\mu)|$ & $>10 \mathrm{GeV},<2.5$ & $>10 \mathrm{GeV},<2.5$ \\
& Cut Conditions & 0 \\
$n_{\ell}$ & 0 & $\leq 4$ \\
$n_{j}$ & $\leq 4$ & $>1500 \mathrm{GeV},<2.4$ \\
$p_{\mathrm{T}}\left(j_{1}\right),\left|\eta\left(j_{1}\right)\right|$ & $>250 \mathrm{GeV},<2.4$ & $>0.4$ \\
$\Delta \phi\left(j, \not \phi_{\mathrm{T}}\right)$ & $>0.4$ & $>1200-2500 \mathrm{GeV}$ \\
$E_{\mathrm{T}}$ & $>250-1000 \mathrm{GeV}$ & \\
\hline \hline
\end{tabular}

the quadruplets contain more dark sector particles than the triplet.

Below we estimate the sensitivity of the monojet $+\mathscr{E}_{\mathrm{T}}$ channel at the SPPC with $\sqrt{s}=100 \mathrm{TeV}$. The signal significance $\mathcal{S}$ can be defined as [45]

$$
\mathcal{S}=\frac{S}{\sqrt{B+(\lambda B)^{2}+(\gamma S)^{2}}},
$$

where $S$ and $B$ are the numbers of signal and background events, respectively, while $\lambda$ and $\gamma$ indicate the systematic uncertainties of the background and the signal, respectively. In order to improve the significance, one needs to perform some efficient cuts. For the monojet $+E_{\mathrm{T}}$ channel, cuts on $E_{\mathrm{T}}$ and the transverse momentum of the leading jet $\left(p_{\mathrm{T}}\left(j_{1}\right)\right)$ are very important. By analyzing the monojet $+E_{\mathrm{T}}$ distributions of the backgrounds and some signal benchmark points (BMPs), we can deduce proper values or intervals of the kinematic variables for cut conditions.

We consider four signal BMPs, whose model parameters are tabulated in Table II. Figures 3(a) and 3(b) demonstrate the differential cross sections and the fractions in each bins for backgrounds and BMPs as functions of $E_{\mathrm{T}}$ at $\sqrt{s}=100 \mathrm{TeV}$. Here we have performed a preselection by requiring $p_{\mathrm{T}}\left(j_{1}\right)>1200 \mathrm{GeV}$ [45]. It is obvious that the distributions of the signals are likely to extend to higher $E_{\mathrm{T}}$ than the backgrounds.

The reconstruction and cut conditions we adopt for SPPC are tabulated in the third column of Table I. In order to efficiently improve the signal significance, we consider six signal regions with different cuts on $E_{\mathrm{T}}$, as listed in Table III. It is difficult to accurately evaluate the systematic uncertainties for future detectors. Here we simply set $\gamma=10 \%$ and $\lambda=1 \%$ [45]. Although these 


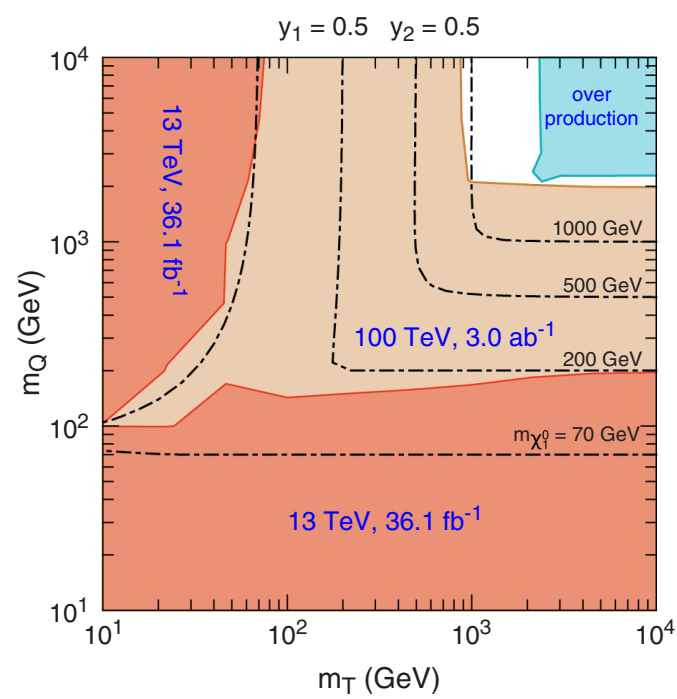

(a) $y_{1}=y_{2}=0.5$

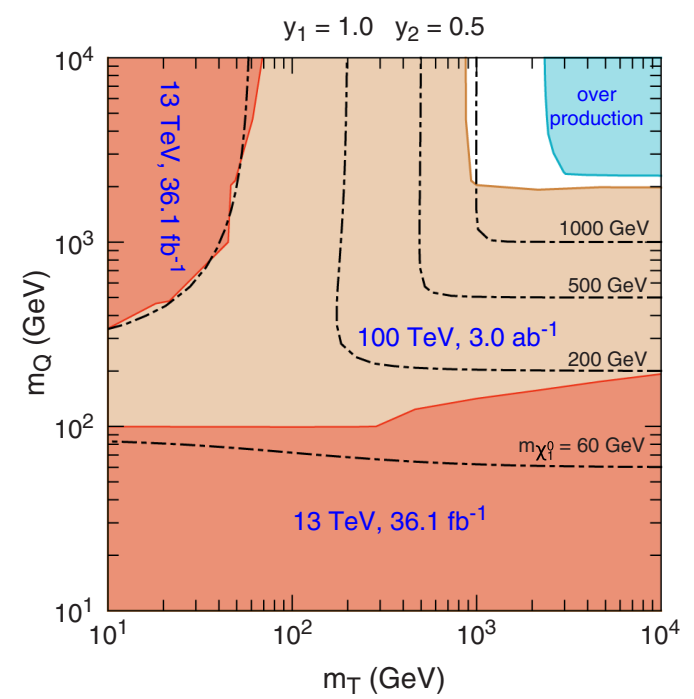

(b) $y_{1}=1.0, y_{2}=0.5$

FIG. 2. LHC constraints and SPPC sensitivities from the monojet $+E_{\mathrm{T}}$ channel in the $m_{T}-m_{Q}$ plane with the fixed Yukawa couplings of $y_{1}=y_{2}=0.5$ (a) and $\left(y_{1}, y_{2}\right)=(1.0,0.5)$ (b). The orange regions are excluded at 95\% C.L. by the ATLAS search at $\sqrt{s}=13$ TeV with a data set of $36.1 \mathrm{fb}^{-1}$ [44]. The canary yellow regions are expected to be excluded at 95\% C.L. at the SPPC with $\sqrt{s}=100 \mathrm{TeV}$ and an integrated luminosity of $3 \mathrm{ab}^{-1}$. The blue regions represent the parameter regions excluded by overproduction of dark matter in the early universe. For reading convenience, contours of $m_{\chi_{1}^{0}}$ are also demonstrated with the dot-dashed lines.

values may be overly optimistic, they provide a benchmark case for estimating the signal significance.

95\% C.L. expected exclusion limits of the monojet $+E_{\mathrm{T}}$ channel at the SPPC with $\sqrt{s}=100 \mathrm{TeV}$ and an integrated luminosity of $3 \mathrm{ab}^{-1}$ are indicated by the canary yellow regions in Fig. 2. Compared with the capability of the LHC, SPPC will improve the search ranges of the mass parameters by an order of magnitude. When a pure triplet (quadruplet) $\chi_{1}^{0}$, the monojet $+E_{\mathrm{T}}^{\prime}$ search at the SPPC could reach up to $m_{\chi_{1}^{0}} \sim 900(2000) \mathrm{GeV}$.

Two Yukawa parameter sets with $y_{1}=y_{2}$ and $y_{1} \neq y_{2}$, i.e., the cases that the custodial symmetry is respected and violated, are considered in Fig. 2(a) and Fig. 2(b). We find that the variation of the Yukawa couplings does not significantly affect the SPPC sensitivity. As we have mentioned in Sec. II B, this is because Dirac masses induced by $y_{1}$ and $y_{2}$ are quite small, compared to TeVscale $m_{T}$ and $m_{Q}$.

In Fig. 2, we also demonstrate the constraints from the observed DM relic density. We utilize the package MadDM

TABLE II. Models parameters of the signal BMPs in the monojet $+E_{\mathrm{T}}$ channel.

\begin{tabular}{lcccc}
\hline \hline & $y_{1}$ & $y_{2}$ & $m_{T} / \mathrm{GeV}$ & $m_{Q} / \mathrm{GeV}$ \\
\hline BMP-a1 & 0.23 & 0.79 & 323 & 473 \\
BMP-a2 & 0.15 & 0.80 & 200 & 700 \\
BMP-a3 & 0.45 & 0.50 & 500 & 600 \\
BMP-a4 & 0.91 & 0.81 & 950 & 860 \\
\hline \hline
\end{tabular}

2.0.6 [68] to calculate the thermal relic density in the TQDM model. All annihilation and coannihilation processes at the freeze-out epoch have been taken into account. The blue regions in Fig. 2 are excluded due to DM overproduction, i.e., the predicted $\Omega_{\chi} h^{2}$ is larger than the observed value 0.1186 [69]. Compared with this constraint, we can see that the SPPC search will be able to explore a very large region in the allowed parameter space.

\section{B. Disappearing track channel}

In some supersymmetric models, such as the anomaly mediated supersymmetry breaking (AMSB) scenario [70,71], the lightest chargino $\tilde{\chi}_{1}^{ \pm}$is nearly mass degenerate with the lightest supersymmetric particle (LSP). The lifetime of chargino can be long enough to travel a distinct distance in the inner detector before decaying into the LSP and a very soft, unlikely detected SM particles, such as pion. Therefore, the track arising from such a long-lived chargino seems to disappear, and only leaves hits in the innermost layers. There would be no hit in the portions of the detector at higher radii, because the LSP passes through the detector without interaction [46]. This is the reason why such a signature is called disappearing tracks.

In the TQDM model, there are three cases that could lead to a mass degeneracy between $\chi_{1}^{ \pm}$and $\chi_{1}^{0}$ with $m_{\chi_{1}^{ \pm}}-m_{\chi_{1}^{0}} \sim$ $\mathcal{O}(100) \mathrm{MeV}$. They would also induce a disappearing tack signal at colliders. As mentioned in Sec. II, a custodial symmetry in the limit of $\left|y_{1}\right|=\left|y_{2}\right|$ would lead to such a mass spectrum. Moreover, when $m_{T} \gg$ $\max \left(m_{Q},\left|y_{1}\right| v,\left|y_{2}\right| v\right)$ or $m_{Q} \gg \max \left(m_{T},\left|y_{1}\right| v,\left|y_{2}\right| v\right)$, the 


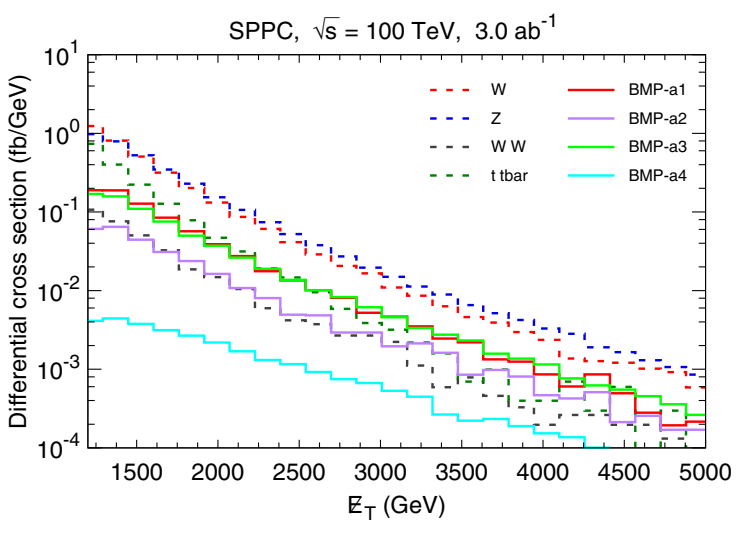

(a) Differential cross sections

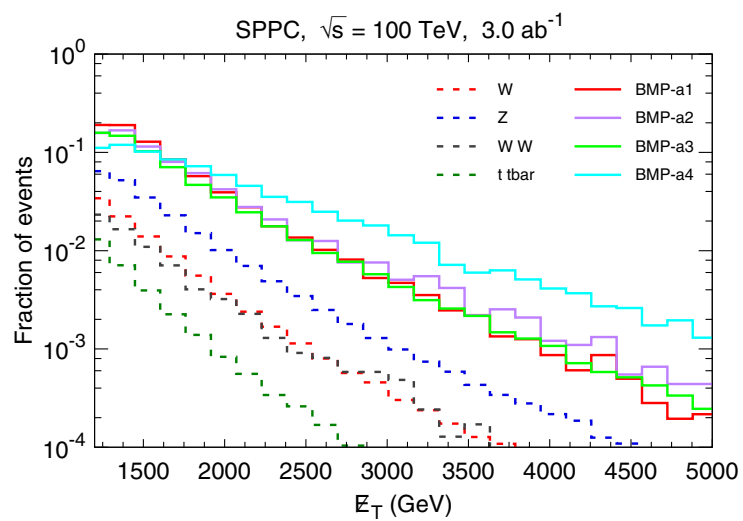

(b) Normalized distributions

FIG. 3. Distributions of differential cross sections (a) and fractions of events (b) as functions of $E_{\mathrm{T}}$ for backgrounds and signal BMPs in the monojet $+E_{\mathrm{T}}$ channel at $\sqrt{s}=100 \mathrm{TeV}$.

quadruplet components or the triplet component in $\chi_{1}^{0}$ is almost dominant. In these two cases, $\chi_{1}^{0}$ is almost degenerate with $\chi_{1}^{ \pm}$in mass even for $y_{1} \neq y_{2}$, because the mixing terms in mass matrices are suppressed by $m_{T}$ or $m_{Q}$. In the following study, we focus on the latter two cases with $m_{T} \ll m_{Q}$ (pure triplet case) and $m_{T} \gg m_{Q}$ (pure quadruplet case) and take $y_{1}=y_{2}=0$ for simplicity.

The current ATLAS search for disappearing tracks [46] is based on $36.1 \mathrm{fb}^{-1}$ of data at $\sqrt{s}=13 \mathrm{TeV}$. The cut conditions are summarized in the second column of Table IV. A critical object for this search is called a pixel tracklet, which contains at least four pixel-detector hits and with no hits in the strip semiconductor tracker and transition radiation tracker detectors. Besides, all these pixel tracklets must not belong to any standard track. By such a definition, pixel tracklets mimics disappearing tracks we are looking for.

Before analyzing the disappearing track signature in the TQDM model, we should check the validity of our simulation and analysis method. We attempt to reproduce the $\sigma_{\text {vis }}$ contour in the $m_{\tilde{\chi}_{1}^{ \pm}}-\tau_{\tilde{\chi}_{1}^{ \pm}}$plane for the AMSB model according to the 95\% C.L. observed limit of $\sigma_{\text {vis }}=$ $0.22 \mathrm{fb}$ given in the ATLAS analysis. Here $\sigma_{\mathrm{vis}}$ is the signal visible cross section after imposing kinematic cuts. From Fig. 4, we can see that our Monte Carlo (MC) result matches with the ATLAS result quit well. It is worth noting that the disappearing condition of this latest ATLAS analysis is different from previous works [45,47]. The previous ATLAS analysis [47] requires the track length of

TABLE III. Inclusive signal regions with different thresholds of $E_{\mathrm{T}}^{\prime}$ in the monojet $+E_{\mathrm{T}}$ channel.

\begin{tabular}{lcccccc}
\hline \hline $\begin{array}{l}\text { Inclusive } \\
\text { signal region }\end{array}$ & IM1 & IM2 & IM3 & IM4 & IM5 & IM6 \\
\hline \hline$E_{\mathrm{T}} \mathrm{GeV}$ & $>1200$ & $>1400$ & $>1600$ & $>1800$ & $>2000$ & $>2500$ \\
\hline \hline
\end{tabular}

unstable charged particles larger than $\sim 30 \mathrm{~cm}$, while much shorter tracks, especially the pixel tracklets, are used in the latest analysis.

Then we study the constraint from the disappearing track search on the TQDM model. The signal events are generated through $p p \rightarrow \chi \chi+$ jets. A leading jet from ISR is required to ensure a significant $E_{\mathrm{T}}$, making the trigger more efficient. For the pure triplet case, the mass splitting

TABLE IV. Reconstruction and cut conditions in the disappearing track channel. The second column is used in the ATLAS analysis at the $13 \mathrm{TeV}$ LHC [46]. The third column is used for evaluating the sensitivity at the $100 \mathrm{TeV}$ SPPC. $j$ with a subscript "top4" means a jet belongs to the four highest- $p_{\mathrm{T}}$ jets. Note that all the terms "tracklets" in the table represent the pixel tracklets.

\begin{tabular}{|c|c|c|}
\hline & $13 \mathrm{TeV}$ LHC & $100 \mathrm{TeV}$ SPPC \\
\hline \multicolumn{3}{|c|}{ Reconstruction Conditions } \\
\hline$p_{\mathrm{T}}(j),|\eta(j)|$ & $>20 \mathrm{GeV},<2.8$ & $>80 \mathrm{GeV},<2.8$ \\
\hline$p_{\mathrm{T}}(e),|\eta(e)|$ & $>10 \mathrm{GeV},<2.47$ & $>10 \mathrm{GeV},<2.47$ \\
\hline$p_{\mathrm{T}}(\mu),|\eta(\mu)|$ & $>10 \mathrm{GeV},<2.7$ & $>10 \mathrm{GeV},<2.7$ \\
\hline Tracklet candidate & $>20 \mathrm{GeV},<2.2$ & $>80 \mathrm{GeV},<2.2$ \\
\hline \multicolumn{3}{|c|}{ Cut Conditions } \\
\hline$n_{\ell}$ & 0 & 0 \\
\hline$n_{j}$ & $\geq 1$ & $\geq 1$ \\
\hline$n_{\text {tracklet }}$ & 1 & 1 \\
\hline$p_{\mathrm{T}}\left(j_{1}\right),\left|\eta\left(j_{1}\right)\right|$ & $>140 \mathrm{GeV},-$ & $\begin{array}{c}>1500 \mathrm{GeV}, \\
<2.4\end{array}$ \\
\hline$\Delta \phi\left(j_{\left[p_{\mathrm{T}}>50 \mathrm{GeV}, \text { top4 }\right]}, \not \not_{\mathrm{T}}\right)$ & $>1$ & $\cdots$ \\
\hline$\Delta \phi\left(j_{\left[p_{\mathrm{T}}>300 \mathrm{GeV}, \text { top } 4\right]}, \not \not_{\mathrm{T}}\right)$ & $\cdots$ & $>1$ \\
\hline Tracklet $p_{\mathrm{T}},|\eta|$ & $\begin{array}{l}\quad>100 \mathrm{GeV} \\
(0.1,1.9),(0.1,1.9)\end{array}$ & $\begin{array}{r}>500 \mathrm{GeV}, \\
(0.1,1.9)\end{array}$ \\
\hline $\begin{array}{l}\Delta R \text { (tracklet, } \\
\left.\quad j_{\left[p_{\mathrm{T}}>50 \mathrm{GeV}\right]}\right)\end{array}$ & $>0.4$ & $\cdots$ \\
\hline $\begin{array}{l}\Delta R(\text { tracklet, } \\
\left.j_{\left[p_{\mathrm{T}}>300 \mathrm{GeV}\right]}\right)\end{array}$ & .. & $>0.4$ \\
\hline$E_{\mathrm{T}}$ & $>140 \mathrm{GeV}$ & $>1500 \mathrm{GeV}$ \\
\hline
\end{tabular}




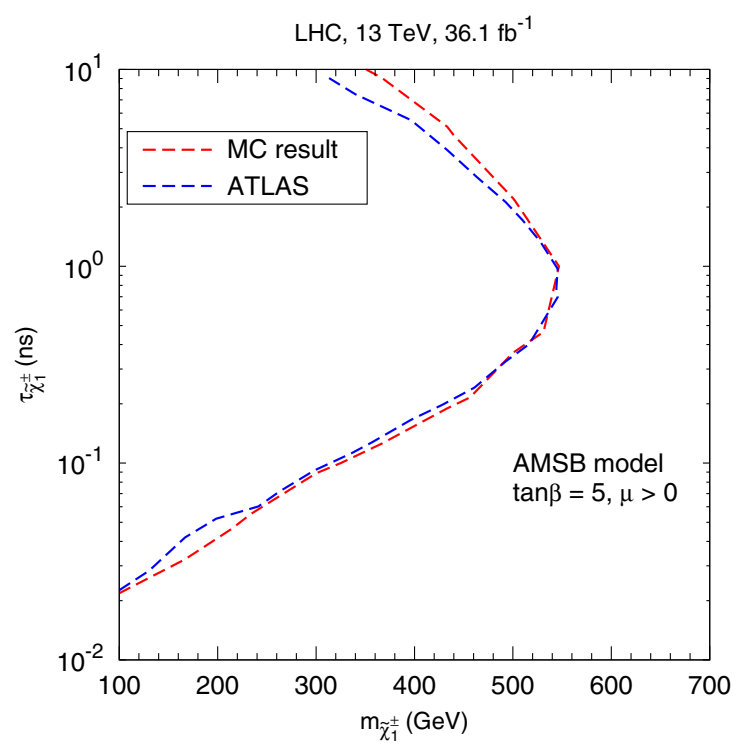

FIG. 4. The comparison between the ATLAS result [46] and our MC result in the disappearing track channel for the $\sigma_{\text {vis }}$ limit at $95 \%$ C.L. in the $m_{\tilde{\chi}_{1}^{ \pm}}-\tau_{\tilde{\chi}_{1}^{ \pm}}$plane for the AMSB model.

between $\chi_{1}^{ \pm}$and $\chi_{1}^{0}$ is $\sim 167 \mathrm{MeV}$. Therefore, the dominant decay process of $\chi_{1}^{ \pm}$is $\chi_{1}^{ \pm} \rightarrow \chi_{1}^{0} \pi^{ \pm}$. For the pure quadruplets case, $\chi_{1}^{ \pm}, \chi_{2}^{ \pm}$, and $\chi^{ \pm \pm}$should be all considered. Because the mass splittings between $\chi^{ \pm \pm}$and $\chi_{1}^{ \pm} / \chi_{2}^{ \pm}$are larger than $800 \mathrm{MeV}, \chi^{ \pm \pm}$decays with a lifetime as short as $\sim 10^{-4} \mathrm{~ns}$, and with almost equal branching ratios $\sim 50 \%$ to $\chi_{1}^{ \pm}$and $\chi_{2}^{ \pm}$. Thus, we only need to consider the lifetimes of $\chi_{1}^{ \pm}$and $\chi_{2}^{ \pm}$for the disappearing track search.

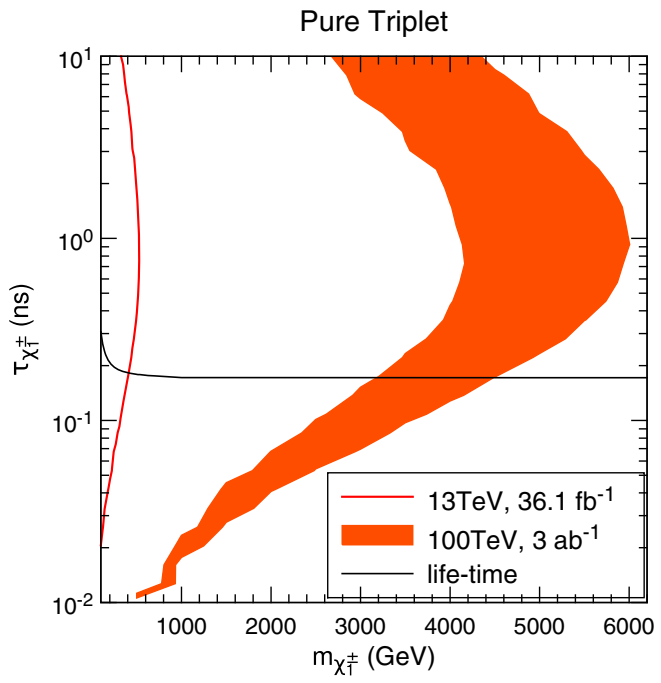

(a) Pure triplet case
The decay widths of the $\chi_{i}^{ \pm} \rightarrow \chi_{j}^{0} \pi^{ \pm}$processes are given by [72]

$$
\begin{aligned}
\Gamma\left(\chi_{i}^{ \pm}\right. & \left.\rightarrow \chi_{j}^{0} \pi^{ \pm}\right) \\
= & \frac{f_{\pi}^{2} G_{\mathrm{F}}^{2}}{4 \pi} \frac{\left|\mathbf{k}_{\pi}\right|}{m_{\chi_{i}^{ \pm}}^{2}}\left\{\left(O_{i j}^{\mathrm{L}}+O_{i j}^{\mathrm{R}}\right)^{2}\right. \\
& \times\left[\left(m_{\chi_{i}^{ \pm}}^{2}-m_{\chi_{j}^{0}}^{2}\right)^{2}-m_{\pi}^{2}\left(m_{\chi_{i}^{ \pm}}-m_{\chi_{j}^{0}}\right)^{2}\right] \\
& \left.+\left(O_{i j}^{\mathrm{L}}-O_{i j}^{\mathrm{R}}\right)^{2}\left[\left(m_{\chi_{i}^{ \pm}}^{2}-m_{\chi_{j}^{0}}^{2}\right)^{2}-m_{\pi}^{2}\left(m_{\chi_{i}^{ \pm}}+m_{\chi_{j}^{0}}\right)^{2}\right]\right\},
\end{aligned}
$$

where $f_{\pi} \simeq 93 \mathrm{MeV}$ is the pion decay constant, and $G_{\mathrm{F}}$ is the Fermi coupling constant. $O_{i j}^{\mathrm{L}}$ and $O_{i j}^{\mathrm{R}}$ are $\chi_{i}^{0} \chi_{j}^{ \pm} W$ couplings and can be read from Eq. (22):

$$
\begin{aligned}
& O_{i j}^{\mathrm{L}}=-\mathcal{N}_{1 i}^{*} \mathcal{C}_{R, 1 j}+\sqrt{2} \mathcal{N}_{2 i}^{*} \mathcal{C}_{R, 2 j}+\frac{\sqrt{6}}{2} \mathcal{N}_{3 i}^{*} \mathcal{C}_{R, 3 j}, \\
& O_{i j}^{\mathrm{R}}=\mathcal{N}_{1 i} \mathcal{C}_{L, 1 j}^{*}+\frac{\sqrt{6}}{2} \mathcal{N}_{2 i} \mathcal{C}_{L, 2 j}^{*}+\sqrt{2} \mathcal{N}_{3 i} \mathcal{C}_{L, 3 j}^{*}
\end{aligned}
$$

$\left|\mathbf{k}_{\pi}\right|$ is the 3-momentum norm of the pion in $\chi_{i}^{ \pm}$rest frame given by

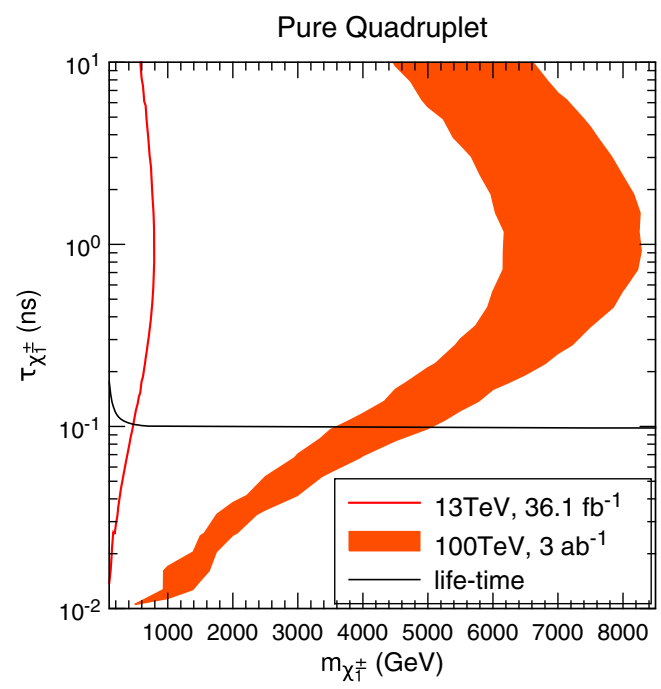

(b) Pure quadruplet case

FIG. 5. $95 \%$ C.L. exclusion limits from the disappearing track channel in the $m_{\chi_{1}^{ \pm}}-\tau_{\chi_{1}^{ \pm}}$plane for the pure triplet (a) and pure quadruplet (b) cases of the TQDM model. The red solid lines represents the current exclusion limit from the ATLAS search based on a data set of $36.1 \mathrm{fb}^{-1}$ at the $13 \mathrm{TeV}$ LHC [46]. The red bands denote the ranges of the expected exclusion limit we estimate for the $100 \mathrm{TeV}$ SPPC search with an integrated luminosity of $3 \mathrm{fb}^{-1}$ by varying a background normalization factor in the range of $0.2-5$. The black solid lines indicate the predicted lifetime of $\chi_{1}^{ \pm}$. 
TABLE V. Information of the signal BMPs in the disappearing track channel.

\begin{tabular}{lcccc}
\hline \hline \multicolumn{5}{c}{ Pure Triplet Case, $m_{Q}=20 \mathrm{TeV}, y_{1}=y_{2}=0$} \\
\hline & BMP-b1 & BMP-b2 & BMP-b3 & BMP-b4 \\
\hline$m_{T} / \mathrm{TeV}$ & 2.0 & 3.0 & 3.5 & 4.0 \\
\hline \hline
\end{tabular}

\begin{tabular}{ccccc}
\hline \multicolumn{5}{c}{ Pure Quadruplet Case, $m_{T}=200 \mathrm{TeV}, y_{1}=y_{2}=0$} \\
\hline & BMP-c1 & BMP-c2 & BMP-c3 & BMP-c4 \\
\hline$m_{Q} / \mathrm{TeV}$ & 1.0 & 2.5 & 4.5 & 5.0 \\
\hline \hline
\end{tabular}

$\left|\mathbf{k}_{\pi}\right|=\frac{1}{2 m_{\chi_{i}^{ \pm}}}\left[\left(m_{\chi_{i}^{ \pm}}^{2}-\left(m_{\chi_{j}^{0}}+m_{\pi}\right)^{2}\right)\left(m_{\chi_{i}^{ \pm}}^{2}-\left(m_{\chi_{j}^{0}}-m_{\pi}\right)^{2}\right)\right]^{1 / 2}$.

The predicted lifetime of $\chi_{1}^{ \pm}$as a function of $m_{\chi_{1}^{ \pm}}$is indicated by the black solid lines in Figs. 5(a) and 5(b) for the pure triplet and pure quadruplet cases, respectively. In the plots, we also show the $95 \%$ C.L. exclusion limit from the ATLAS search as the red lines. When deriving these limit curves, the lifetime of $\chi_{1}^{ \pm}$for each value of $m_{\chi_{1}^{ \pm}}$ is treated as a free parameter. Therefore, the intersections between the black and red lines provide lower limits on $m_{\chi_{1}^{ \pm}}$. For the pure triplet (quadruplet) case, $m_{\chi_{1}^{ \pm}} \lesssim$ $410(472) \mathrm{GeV}$ is excluded at $95 \%$ C.L. Such a constraint is much stronger than the current monojet $+E_{\mathrm{T}}$ constraint, but it should be noted that the disappearing track channel is only sensitive to very restricted parameter regions where $m_{\chi_{1}^{ \pm}}-m_{\chi_{1}^{0}} \lesssim 170 \mathrm{MeV}$.

Below we discuss the prospect of the disappearing track channel at the $100 \mathrm{TeV}$ SPPC. The SM backgrounds are mainly contributed by the $W+$ jets and $t \bar{t}+$ jets processes. Nonetheless, there are also three kinds of fake pixel tracklets that could contribute to the backgrounds [46]:

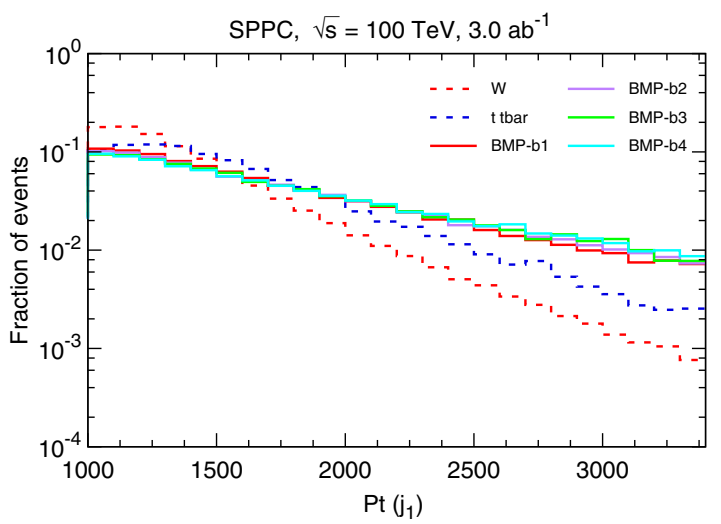

(a) $p_{\mathrm{T}}\left(j_{1}\right)$ distributions in the pure triplet case
(1) A hadron undergoes a hard scattering with the inner detector and is not recognized as belonging to the same track.

(2) A lepton emitting a hard photon could be identified as a disappearing tracklet.

(3) A random combination of hits can be created by different nearby particles.

All these known and other potentially unknown detector effects make us impossible to accurately simulate the backgrounds at the SPPC. Instead, we can perform a simple estimation by rescaling the number of background events at the $13 \mathrm{TeV}$ LHC [46], according to the event rates of the $W+$ jets and $t \bar{t}+$ jets backgrounds at the SPPC and LHC $[12,45,48,49]$.

We choose several BMPs for the pure triplet and pure quadruplet cases, as listed in Table V. Figure 6 shows the normalized distributions of $p_{\mathrm{T}}\left(j_{1}\right)$ and $E_{\mathrm{T}}^{t}$ for the backgrounds and signal BMPs at $\sqrt{s}=100 \mathrm{TeV}$. According to these distributions, the cut thresholds for $p_{\mathrm{T}}\left(j_{1}\right)$ and $E_{\mathrm{T}}$ can both be chosen to be $1500 \mathrm{GeV}$. We find that these thresholds are useful for both the pure triplet and quadruplet cases. After applying all the cut conditions listed in the third column of Table IV, the expected background event number would be $\sim 1$ with an integrated luminosity of $3 \mathrm{ab}^{-1}$. However, this number may be underestimated. In order to take into account the uncertainty in the background calculation, we adopt a range for the background estimation by rescaling this number by a factor of $0.2-5$.

The expected 95\% C.L. exclusion limits at the SPPC are given as the red bands in Fig. 5, according to the rescaling factor varying within $20 \%-500 \%$. In the pure triplet case, the SPPC search could reach up to $m_{\chi_{1}^{ \pm}} \lesssim 3.2-4.5 \mathrm{TeV}$. In the pure quadruplets, the lower limit of $m_{\chi_{1}^{ \pm}}$would be raised to 3.5-5.2 TeV. Thus, the disappearing track channel at the SPPC has the potential to explore the whole range of $m_{\chi_{1}^{0}}$ allowed by the observed relic abundance for these two special cases.

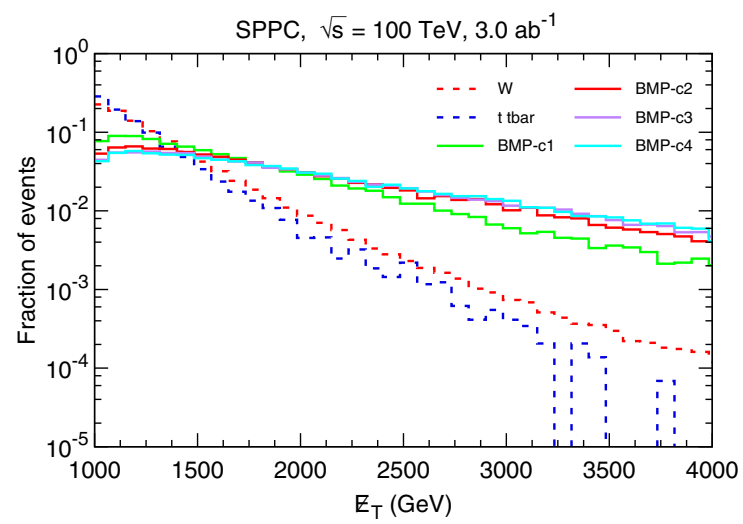

(b) $\mathbb{E}_{\mathrm{T}}$ distributions in the pure quadruplet case

FIG. 6. Normalized distributions of $p_{\mathrm{T}}\left(j_{1}\right)$ in the pure triplet case (a) and $E_{\mathrm{T}}$ in the pure quadruplet case (b) at $\sqrt{s}=100 \mathrm{TeV}$. The dashed and solid lines represent the distributions for the backgrounds and signal BMPs, respectively. 
TABLE VI. Cut conditions for the $2 \ell+0$ jets $+E_{\mathrm{T}}$ inclusive signal regions. The number written in brackets is used for the $100 \mathrm{TeV}$ SPPC, while other numbers are used in the ATLAS analysis at the $13 \mathrm{TeV}$ LHC [51].

\begin{tabular}{lcc}
\hline \hline \multicolumn{3}{c}{$2 \ell+0$ jets $+\not E_{\mathrm{T}}$} \\
\hline$m_{\mathrm{T} 2} / \mathrm{GeV}$ & $m_{\ell \ell} / \mathrm{GeV}$ & Bin Order \\
\hline$>100$ & $>111$ & 1 \\
$>130$ & $>300(>200)$ & 2 \\
$>100$ & $\cdots$ & 3 \\
$>150$ & $\cdots$ & 4 \\
$>200$ & $\cdots$ & 5 \\
$>300$ & $\cdots$ & 6 \\
\hline \hline
\end{tabular}

TABLE VII. Cut conditions for the $2 \ell+$ jets $+E_{\mathrm{T}}$ inclusive signal regions. $j_{1}$ and $j_{3}$ denote the jets with the highest and third highest $p_{\mathrm{T}}$, respectively. $Z$ and $W$ mean the $Z$ and $W$ bosons constructed by the two leptons $(\ell \ell)$ and the two jets $(j j)$, respectively. The numbers in brackets are used for the $100 \mathrm{TeV}$ SPPC, while others are used in the ATLAS analysis at the $13 \mathrm{TeV}$ LHC [51].

\begin{tabular}{|c|c|c|c|c|}
\hline \multicolumn{5}{|c|}{$2 \ell+$ jets $+E_{\mathrm{T}}$ Signal Regions } \\
\hline Bin Order & 7 & 8 & $9 *$ & $10 *$ \\
\hline$n_{\text {light-jets }}$ & $\geq 2$ & $\geq 2$ & 2 & $3-5$ \\
\hline$m_{\ell \ell} / \mathrm{GeV}$ & $81-101$ & 81-101 & $81-101$ & $86-96$ \\
\hline$m_{j j} / \mathrm{GeV}$ & $70-100$ & $70-100$ & $70-90$ & $70-90$ \\
\hline$E_{\mathrm{T}} \mathrm{GeV}$ & $>150$ & $>250$ & $>100$ & $>100$ \\
\hline$p_{\mathrm{T}}^{\mathrm{Z}} / \mathrm{GeV}$ & $>80(>125)$ & $>80(>125)$ & $>60$ & $>40$ \\
\hline$p_{\mathrm{T}}^{W} / \mathrm{GeV}$ & $>100(>130)$ & $>100(>130)$ & & \\
\hline$m_{\mathrm{T} 2} / \mathrm{GeV}$ & $>100$ & $>100$ & & \\
\hline$\Delta R_{j j}$ & $<1.5$ & $<1.5$ & & $<2.2$ \\
\hline$\Delta R_{\ell \ell}$ & $<1.8$ & & & \\
\hline$\Delta \phi\left(\not \not{ }_{\mathrm{T}}, Z\right)$ & & & $<0.8$ & \\
\hline$\Delta \phi\left(\not \not_{\mathrm{T}}, W\right)$ & $0.5-3.0$ & $0.5-3.0$ & $>1.5$ & \\
\hline$E_{\mathrm{T}}^{\prime} / p_{\mathrm{T}}^{Z}$ & & & $0.6-1.6$ & \\
\hline$\not E_{\mathrm{T}} / p_{\mathrm{T}}^{W}$ & & & $<0.8$ & \\
\hline$\Delta \phi\left(\not \not{ }_{\mathrm{T}}, j_{1}\right)$ & & & $>2.6$ & \\
\hline$|\eta(Z)|$ & & & $<1.6$ & \\
\hline$p_{\mathrm{T}}^{j_{3}} / \mathrm{GeV}$ & & & $<1.6$ & \\
\hline
\end{tabular}

\section{Multilepton $+E_{\mathrm{T}}$ channel}

If the kinematics is allowed, charged and heavier neutral fermions in the dark sector would decay into leptonic states via real or virtual $W$ and $Z$ bosons, leading to detectable signals in the multilepton $+E_{\mathrm{T}}$ channel. This channel has been widely used to explore SUSY models [51,52,73,74]. In this subsection, we just focus on the final state containing two or three charged leptons associated with a large $E_{\mathrm{T}}$.

In our simulation, the signal events come from $p p \rightarrow$ $\chi \chi+$ jets. Main SM backgrounds arise from $W W+$ jets, $W Z+$ jets, $Z Z+$ jets, and $t \bar{t}+$ jets production processes. We have compared our MC results for SM backgrounds with the ATLAS MC results [75], and find that they match with each other very well.

We used the latest ATLAS analysis [51] as our primary reference for analyzing the multilepton $+E_{\mathrm{T}}^{\prime}$ channel. This channel can be categorized into three subchannels according to the numbers of leptons and jets in the final state, i.e., the $2 \ell+0$ jets $+E_{\mathrm{T}}, 2 \ell+$ jets $+E_{\mathrm{T}}^{\prime}, 3 \ell+E_{\mathrm{T}}$ channels. There are some common reconstruction conditions for these channels:

(i) All jets must have $p_{\mathrm{T}}>20 \mathrm{GeV}$ and $|\eta|<2.8$.

(ii) Baseline electrons are required to have $p_{\mathrm{T}}>20 \mathrm{GeV}$ and $|\eta|<2.47$.

(iii) Baseline muons are required to have $p_{\mathrm{T}}>20 \mathrm{GeV}$ and $|\eta|<2.5$.

(iv) Central light-jets, which are tagged as $b$-jets, are required to have $p_{\mathrm{T}}>60 \mathrm{GeV}$ and $|\eta|<2.4$.

(v) Central $b$-jets are required to have $p_{\mathrm{T}}>20 \mathrm{GeV}$ and $|\eta|<2.4$.

In the $2 \ell+0$ jets $+E_{\mathrm{T}}$ channel, the leading and subleading leptons are required to have $p_{\mathrm{T}}>25 \mathrm{GeV}$ and $p_{\mathrm{T}}>$ $20 \mathrm{GeV}$, respectively, and the signal events should not contain any central light-jet or central $b$-jet. Other cut conditions for the signal regions in the three channels defined in the ATLAS analysis are listed in Tables VI, VII, andVIII, where the numbers in brackets are the improved values we choose for the $100 \mathrm{TeV}$ SPPC.

Note that in the $2 \ell+0$ jets $+E_{\mathrm{T}}$ channel, the kinematic variable $m_{\mathrm{T} 2}[76-78]$ is utilized instead of $E_{\mathrm{T}}$ in order to effectively suppress backgrounds. Thus, this channel is

TABLE VIII. Cut conditions for the $3 \ell+E_{\mathrm{T}}$ binned signal regions in the ATLAS analysis at the $13 \mathrm{TeV}$ LHC [51]. $\ell_{3}$ denotes the lepton with the third highest $p_{\mathrm{T}}$, while $j_{1}$ denotes the jet with the highest $p_{\mathrm{T}}$.

\begin{tabular}{|c|c|c|c|c|c|c|c|}
\hline \multicolumn{8}{|c|}{$3 \ell+E_{\mathrm{T}}$ Binned Signal Region } \\
\hline$m_{\mathrm{SFOS}} / \mathrm{GeV}$ & $\not E_{\mathrm{T}} / \mathrm{GeV}$ & $p_{\mathrm{T}}^{\ell_{3}} / \mathrm{GeV}$ & $n_{\text {light-jets }}$ & $m_{\mathrm{T}}^{\min } / \mathrm{GeV}$ & $p_{\mathrm{T}}^{\ell \ell \ell} / \mathrm{GeV}$ & $p_{\mathrm{T}}^{j_{1}} / \mathrm{GeV}$ & bin order \\
\hline $81.2-101.2$ & $60-120$ & & 0 & $>110$ & & & 11 \\
\hline $81.2-101.2$ & $120-170$ & & 0 & $>110$ & & & 12 \\
\hline $81.2-101.2$ & $>170$ & & 0 & $>110$ & & & 13 \\
\hline $81.2-101.2$ & $120-200$ & & $\geq 1$ & $>110$ & $<120$ & $>70$ & 14 \\
\hline $81.2-101.2$ & $>200$ & & $\geq 1$ & $110-160$ & & & 15 \\
\hline $81.2-101.2$ & $>200$ & $>35$ & $\geq 1$ & $>160$ & & & 16 \\
\hline
\end{tabular}




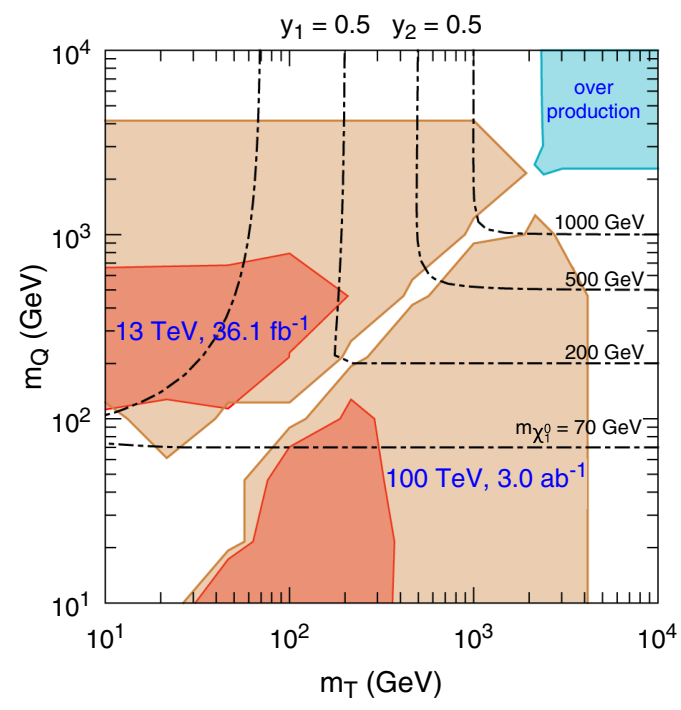

(a) $y_{1}=y_{2}=0.5$

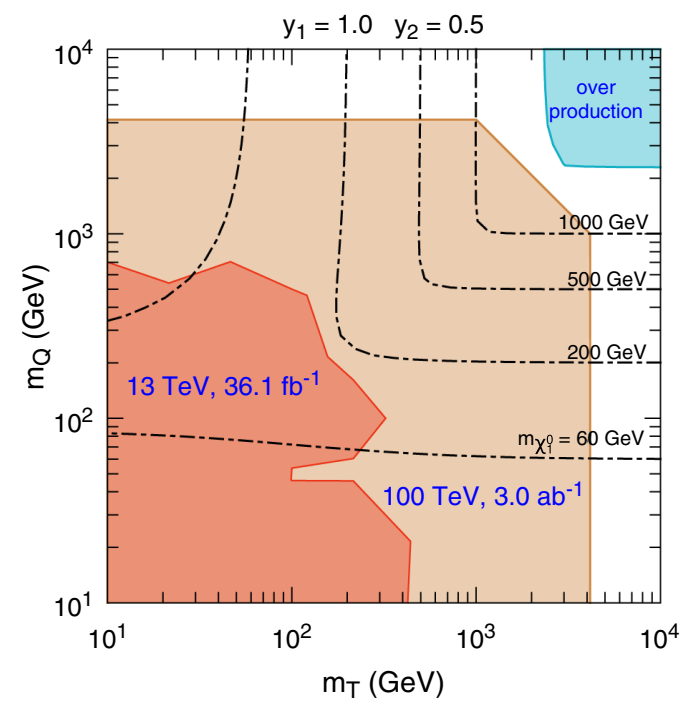

(b) $y_{1}=1.0, y_{2}=0.5$

FIG. 7. LHC Constraints and SPPC sensitivities from the multilepton $+\mathscr{E}_{\mathrm{T}}$ channel in the $m_{T}-m_{Q}$ plane with the fixed Yukawa couplings of $y_{1}=y_{2}=0.5$ (a) and $\left(y_{1}, y_{2}\right)=(1.0,0.5)$ (b). The orange regions are excluded at 95\% C.L. by the ATLAS search at the $13 \mathrm{TeV}$ LHC with a data set of $36.1 \mathrm{fb}^{-1}$ [51], while the canary yellow regions are expected to be excluded at $95 \%$ C.L. by the $100 \mathrm{TeV}$ SPPC with an integrated luminosity of $3 \mathrm{ab}^{-1}$. The blue regions are excluded because of overproduction of dark matter in the early universe.

sensitive to signal processes like $\chi_{2}^{+} \chi_{2}^{-} \rightarrow W^{+}\left(\rightarrow \ell^{+} \nu\right)$ $W^{-}\left(\rightarrow \ell^{\prime-} \bar{\nu}\right) \chi_{1}^{0} \chi_{1}^{0}$. In the $2 \ell+$ jets $+E_{\mathrm{T}}^{\prime}$ channel, two same-flavor opposite-sign (SFOS) leptons are used to reconstruct a $Z$ boson, while two jets are used to reconstruct a $W$ boson. The cut conditions utilize several kinematic variables related to the reconstructed $Z$ and $W$ bosons. Such a channel is useful for searching signals like $\chi_{2}^{0} \chi_{2}^{ \pm} \rightarrow Z\left(\rightarrow \ell^{+} \ell^{-}\right) W^{ \pm}(\rightarrow j j) \chi_{1}^{0} \chi_{1}^{0}$. In the $3 \ell+E_{\mathrm{T}}$ channel, a $Z$ boson is also reconstructed via two SFOS leptons, and the transverse mass $m_{\mathrm{T}}$, which should not be confused with the parameter $m_{T}$ in the TQDM model, is utilized for suppressing backgrounds. This channel would be sensitive to signals like $\chi_{2}^{0} \chi_{2}^{ \pm} \rightarrow Z\left(\rightarrow \ell^{+} \ell^{-}\right) W^{ \pm}(\rightarrow \ell \nu) \chi_{1}^{0} \chi_{1}^{0}$.

The constraint from the multilepton $+E_{\mathrm{T}}$ channel on the TQDM model are shown in Fig. 7 for two Yukawa parameter sets of $y_{1}=y_{2}=0.5$ and $y_{1}=1$ and $y_{2}=0.5$. The orange regions are excluded at $95 \%$ C.L. by the ATLAS search at $\sqrt{s}=13 \mathrm{TeV}$ with $36.1 \mathrm{fb}^{-1}$ of data. In the case of $y_{1}=y_{2}=0.5$, the custodial symmetry is respected, leading to a compressed particle spectrum in the regions with $m_{T} \sim m_{Q}$, small $m_{T}$, or small $m_{Q}$. This means that the leptons from dark sector fermion decays would not be energetic. As a result, the multilepton $+E_{\mathrm{T}}$ channel can hardly explore these parameter regions. In the case of $y_{1}=1.0$ and $y_{2}=0.5$, such parameter regions do not appear.

Then we investigate the SPPC sensitivity. Four BMPs are adopted for studying cut thresholds, as listed in Table IX. In Fig. 8, distributions for the backgrounds and signal BMPs are presented. Here we demonstrate the normalized distributions of two kinematic variables: the invariant mass of the lepton pair $m_{l l}$ for the $2 \ell+$ 0jets $+E_{\mathrm{T}}$ channel and the transverse momentum of the reconstructed $Z$ boson $p_{\mathrm{T}}^{Z}$ for the $2 \ell+$ jets $+E_{\mathrm{T}}^{t}$ channel. Based on such distributions, we choose the cut thresholds of $m_{l l}$ and $p_{\mathrm{T}}^{Z}$ to be 200 and $125 \mathrm{GeV}$, respectively. Other cut conditions can be found in Tables VI and VII.

The canary yellow regions in Fig. 7 are expected to be excluded at $95 \%$ C.L. by the multilepton $+E_{\mathrm{T}}$ search at the $100 \mathrm{TeV}$ SPPC with an integrated luminosity of $3 \mathrm{ab}^{-1}$. We find that such a search would probe the parameter regions up to $m_{T} \sim 4 \mathrm{TeV}$ and $m_{Q} \sim 4 \mathrm{TeV}$. But this channel seems less powerful than the monojet $+E_{\mathrm{T}}$ channel.

\section{CEPC SEARCHES}

With a collision energy of $\sqrt{s} \sim 240-250 \mathrm{GeV}$ and an integrated luminosity of $5 \mathrm{ab}^{-1}$, more than one million Higgs bosons will be produced at the CEPC [38]. The CEPC has a powerful capability to measure the detailed properties of the Higgs boson and explore BSM models

TABLE IX. Information of the signal BMPs in the multilepton $+E_{\mathrm{T}}$ channel.

\begin{tabular}{lcccr}
\hline \hline & $y_{1}$ & $y_{2}$ & $m_{T} / \mathrm{GeV}$ & $m_{Q} / \mathrm{GeV}$ \\
\hline BMP-d1 & 0.5 & 0.5 & 10.0 & 1000.0 \\
BMP-d2 & 0.5 & 0.5 & 215.4 & 464.2 \\
BMP-d3 & 0.5 & 0.5 & 464.2 & 100.0 \\
BMP-d4 & 0.5 & 0.5 & 464.2 & 1000.0 \\
\hline \hline
\end{tabular}




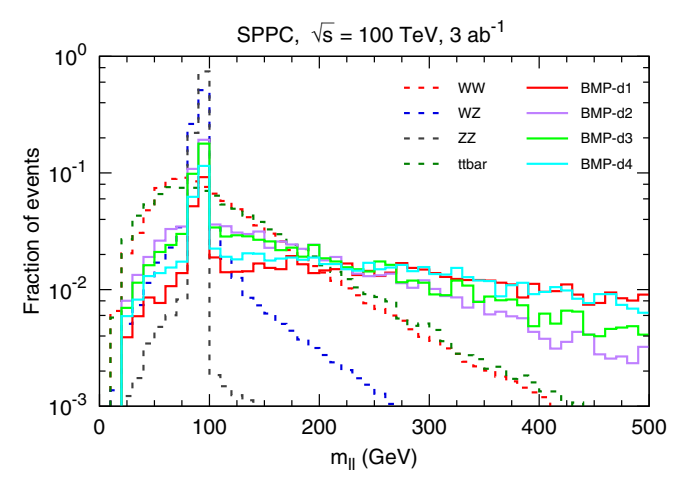

(a) $m_{\ell \ell}$ distributions for $2 \ell+0$ jets $+\mathbb{E}_{\mathrm{T}}$

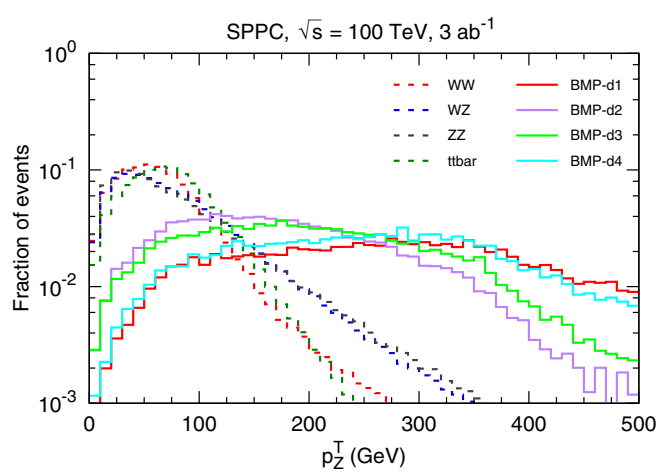

(b) $p_{\mathrm{T}}^{Z}$ distributions for $2 \ell+$ jets $+\mathbb{E}_{\mathrm{T}}$

FIG. 8. Normalized distributions of $m_{\ell \ell}$ in the $2 \ell+0 \mathrm{jets}+\mathscr{E}_{\mathrm{T}}$ final state (a) and $p_{\mathrm{T}}^{Z}$ in the $2 \ell+$ jets $+\mathscr{E}_{\mathrm{T}}$ final state $(\mathrm{b})$ at $\sqrt{s}=100 \mathrm{TeV}$. The dashed and solid lines represent the distributions for the backgrounds and signal BMPs, respectively.

through precision measurements. In this section, we study loop effects on CEPC Higgs measurements induced by dark sector fermions in the TQDM model.

\section{A. $e^{+} e^{-} \rightarrow Z h$ production}

The leading production process of the SM Higgs boson at the CEPC is the $Z h$ associated production $e^{+} e^{-} \rightarrow Z h$. The EW corrections to its cross section $\sigma_{Z h}$ at the next-toleading order (NLO) in the SM can be found in [79-81]. Recently, the mixed QCD-EW $\mathcal{O}\left(\alpha \alpha_{s}\right)$ corrections to $\sigma_{Z h}$ $[82,83]$ and the ISR effects [84] have also been studied. For a data set of $5 \mathrm{ab}^{-1}$, the relative precision of the $\sigma_{Z h}$ measurement would reach down to $\Delta \sigma_{Z h} / \sigma_{Z h} \sim 0.5 \%$.

With such a high precision, new physics effects through loop corrections may manifest. As the dark sector fermions in the TQDM model couple to both the Higgs boson and the EW gauge bosons, it is worth investigating their oneloop correction to $\sigma_{Z h}$. We utilize the packages FeynArts 3.9 [85], FormCalc 9.4 [86] and LoopTools 2.13 [87] to calculate this correction at $\sqrt{s}=240 \mathrm{GeV}$. We adopt the on-shell renormalization scheme and neglect the mass and Yukawa coupling of the electron. The deviation of $\sigma_{Z h}$ from the SM prediction can be expressed as

$$
\Delta \sigma / \sigma_{0}=\left|\sigma-\sigma_{0}\right| / \sigma_{0} .
$$

Here $\sigma_{0}=\sigma_{\mathrm{SM}-\mathrm{LO}}+\Delta \sigma_{\mathrm{SM}-\mathrm{NLO}}$ is the $\mathrm{SM}$ prediction including the leading-order cross section $\sigma_{\mathrm{SM}-\mathrm{LO}}$ and the NLO correction $\Delta \sigma_{\mathrm{SM} \text {-NLO }} \sigma=\sigma_{0}+\Delta \sigma_{\mathrm{TQ}-\mathrm{NLO}}$ involves the NLO contribution from the TQDM model, $\Delta \sigma_{\mathrm{TQ}-\mathrm{NLO}}$. If the predicted $\Delta \sigma / \sigma_{0}$ is larger than $0.5 \%$, the CEPC measurement should be able to probe such an effect of the TQDM model.

We calculate $\Delta \sigma / \sigma_{0}$ for four benchmark cases, as shown in Fig. 9. For each case, we fix two parameters in $\left(m_{T}, m_{Q}, y_{1}, y_{2}\right)$ and vary the other two. The colored regions corresponding to $\Delta \sigma / \sigma_{0}>0.5 \%$ may be explored by the CEPC measurement, while the gray regions are beyond its capability. The dot-dashed lines represent the mass of $\chi_{1}^{0}$.

In Figs. 9(a) and 9(b), the Yukawa couplings are fixed to be $y_{1}=y_{2}=0.5$ and $\left(y_{1}, y_{2}\right)=(1.0,0.5)$, respectively. We can see that the CEPC would explore up to $m_{\chi_{1}^{0}} \sim$ 275-300 GeV for these two cases. When $m_{T} \gtrsim 100 \mathrm{GeV}$ and $m_{Q} \gtrsim 300 \mathrm{GeV}$, all dark sector fermions become heavy, suppressing the Higgs effective interactions with the photon and $Z$. Thus, the corrections in these region would not be significant.

It is promising to search the loop effects on $\sigma_{z h}$ for small $m_{T}$ and $m_{Q}$. Thus, we fix $\left(m_{T}, m_{Q}\right)$ to be $(100,400) \mathrm{GeV}$ and $(400,200) \mathrm{GeV}$ in Figs. 9(c) and 9(d), respectively, to examine how $\Delta \sigma / \sigma_{0}$ varies with $y_{1}$ and $y_{2}$. In these two cases, $\chi_{1}^{0}$ is dominated by either the triplet or the quadruplets. We can see that $\Delta \sigma / \sigma_{0}>0.5 \%$ almost holds in the whole $y_{1}-y_{2}$ plane.

Note that the variation of $\Delta \sigma / \sigma_{0}$ in all these plots looks quite complicated. This is mainly due to the threshold effect. For instance, in the regions with $m_{\chi_{i}^{0}}+m_{\chi_{j}^{0}} \sim m_{Z}$, $m_{\chi_{i}^{0}}+m_{\chi_{j}^{-}} \sim m_{W}, m_{\chi_{i}^{0}}+m_{\chi_{j}^{-}}\left(m_{\chi_{j}^{0}}\right) \sim \sqrt{s}$ and so on, the propagators of dark sector fermions meet some poles, and their contributions change dramatically. In order to more explicitly analyze such a effect, we plot the contours indicating some threshold conditions in Fig. 10. Comparing Figs. 9(a) and 9(c) with Fig. 10, we can find that most of the $\Delta \sigma / \sigma_{0}$ structures match these contours.

\section{B. $h \rightarrow \gamma \gamma$ decay}

In the SM, the Higgs boson decay into diphoton is induced by loops of the $W$ boson and heavy charged fermions. The deviation of the $h \rightarrow \gamma \gamma$ partial width $\Gamma_{\gamma \gamma}$ from the SM prediction $\Gamma_{\gamma \gamma}^{\mathrm{SM}}$ can be characterized by a ratio $\kappa_{\gamma} \equiv \Gamma_{\gamma \gamma} / \Gamma_{\gamma \gamma}^{\mathrm{SM}}$. The CEPC can measure this ratio with a high precision of $4.7 \%$ for an integrated luminosity of $5 \mathrm{ab}^{-1}$ [38]. In the TQDM model, the singly charged fermions $\chi_{i}^{ \pm}$ couple to both the Higgs boson and the photon, and hence 


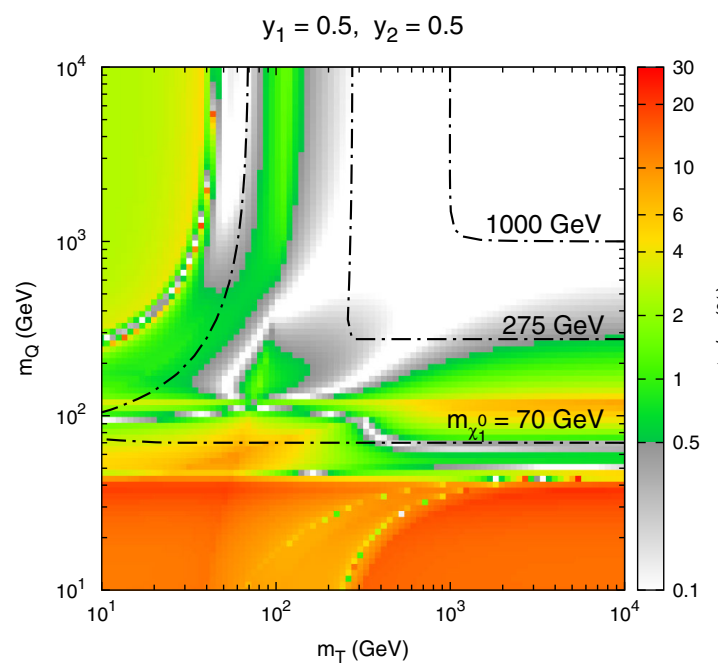

(a) $y_{1}=y_{2}=0.5$

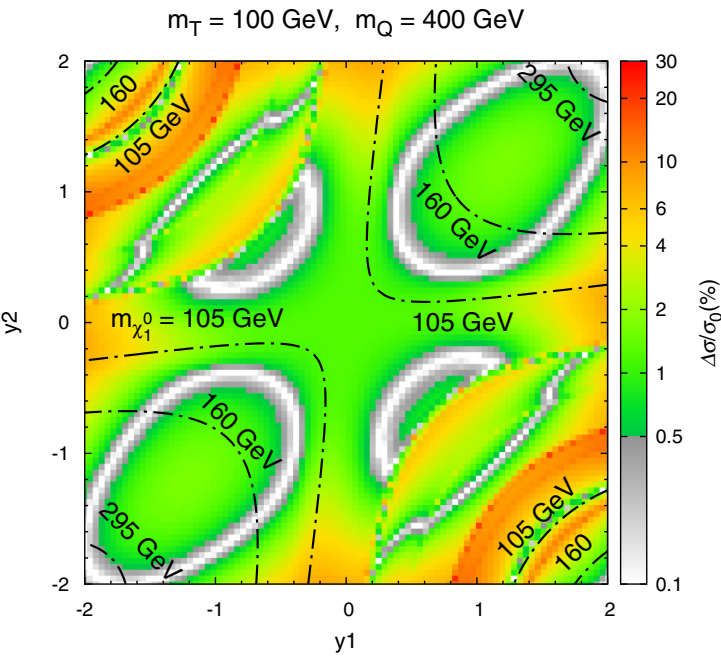

(c) $m_{T}=100 \mathrm{GeV}, m_{Q}=400 \mathrm{GeV}$

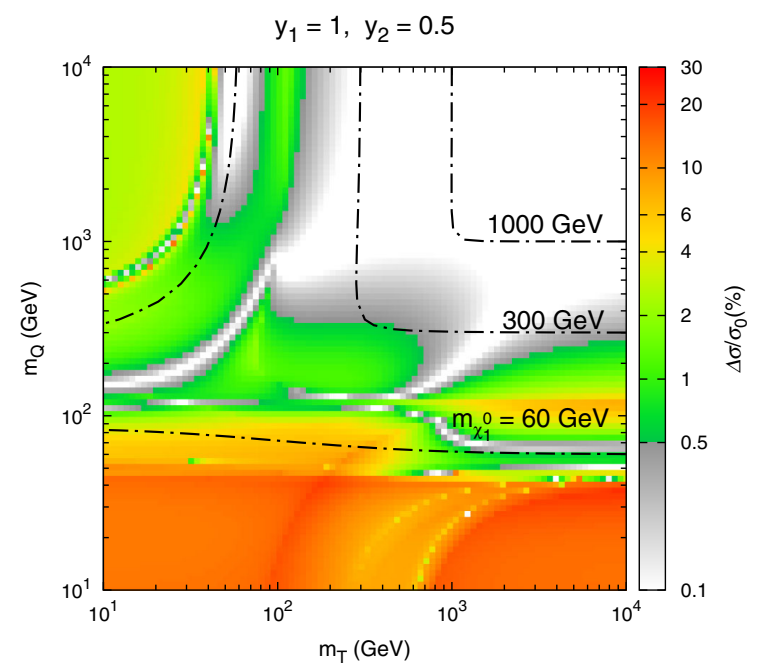

(b) $y_{1}=1.0, y_{2}=0.5$

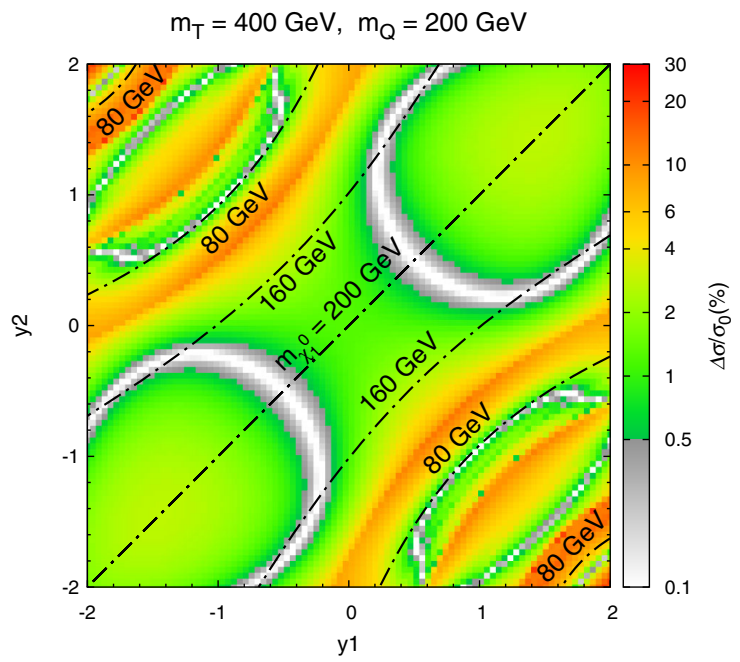

(d) $m_{T}=400 \mathrm{GeV}, m_{Q}=200 \mathrm{GeV}$

FIG. 9. Heat maps for the relative deviation of the $e^{+} e^{-} \rightarrow Z h$ cross section $\Delta \sigma / \sigma_{0}$ predicted in the TQDM model. The top and bottom panels show the results in the $m_{T}-m_{Q}$ and $y_{1}-y_{2}$ planes with the other two parameters fixed, respectively. Colored and gray regions correspond to $\Delta \sigma / \sigma_{0}>0.5 \%$ and $<0.5 \%$, respectively. Dot-dashed lines denote the contour of $m_{\chi_{1}^{0}}$.

modify the value of $\kappa_{\gamma}$ from 1 . Therefore, the measurement of $h \rightarrow \gamma \gamma$ could be useful for exploring the TQDM model.

Note that the doubly charged fermion $\chi^{ \pm \pm}$, which is only constructed by the quadruplets, does not contribute to $\Gamma_{\gamma \gamma}$. This is because an $\mathrm{SU}(2)_{\mathrm{L}}$ invariant Yukawa term $\sim$ QTH must be built from a triplet and a quadruplet. The $h \rightarrow \gamma \gamma$ partial decay width can be cast into the form of $[36,88-90]$

$$
\begin{aligned}
\Gamma_{\gamma \gamma}= & \frac{G_{\mathrm{F}} \alpha^{2} m_{h}^{3}}{128 \sqrt{2} \pi^{3}} \mid \sum_{f} N_{c} Q_{f}^{2} A_{1 / 2}\left(\tau_{f}\right)+A_{1}\left(\tau_{W}\right) \\
& +\left.\sum_{i} \frac{G_{h, i i} v}{m_{\chi_{i}^{ \pm}}} A_{1 / 2}\left(\tau_{\chi_{i}^{ \pm}}\right)\right|^{2}
\end{aligned}
$$

where $\alpha$ is the fine-structure constant, $N_{c}$ is the color factor, $Q_{f}$ is the electric charge of an SM fermion $f$, and $G_{h, i i}$ is the $\chi_{i}^{-} \chi_{i}^{+} h$ coupling, which can be read from Eq. (19):

$$
\begin{aligned}
G_{h, i i}= & \operatorname{Re}\left(-\frac{y_{1}}{\sqrt{2}} \mathcal{C}_{\mathrm{L}, 2 i} \mathcal{C}_{\mathrm{R}, 1 i}+\frac{y_{1}}{\sqrt{6}} \mathcal{C}_{\mathrm{L}, 1 i} \mathcal{C}_{\mathrm{R}, 2 i}+\frac{y_{2}}{\sqrt{6}} \mathcal{C}_{\mathrm{L}, 3 i} \mathcal{C}_{\mathrm{R}, 1 i}\right. \\
& \left.-\frac{y_{2}}{\sqrt{2}} \mathcal{C}_{\mathrm{L}, 1 i} \mathcal{C}_{\mathrm{R}, 3 i}\right)
\end{aligned}
$$

The first two terms between the vertical bars are SM contributions, while the third term comes from the TQDM model. $A_{1 / 2}$ and $A_{1}$ are the form factors for spin-1/2 and spin-1 particles: 


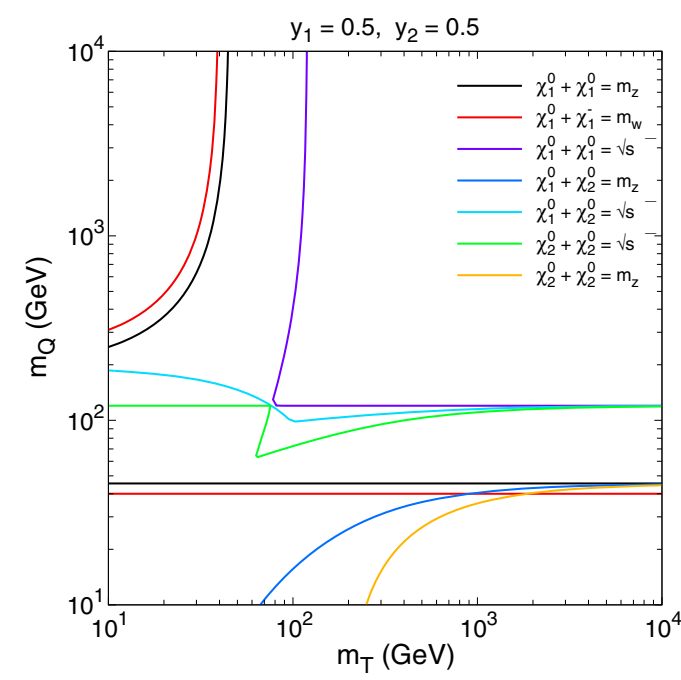

(a) $y_{1}=0.5, y_{2}=0.5$

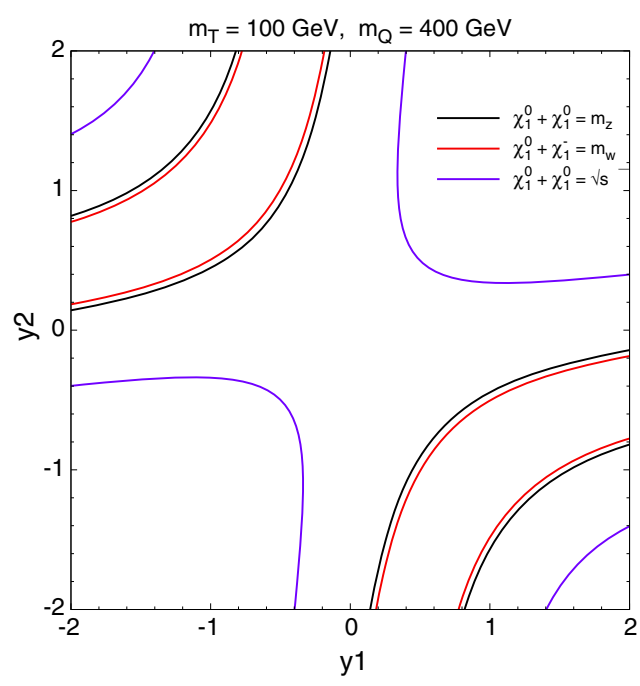

(b) $m_{T}=100 \mathrm{GeV}, m_{Q}=400 \mathrm{GeV}$

FIG. 10. Contours for threshold conditions. The left and right panels correspond to the parameter sets in Fig. 9(a) and Fig. 9(c), respectively.

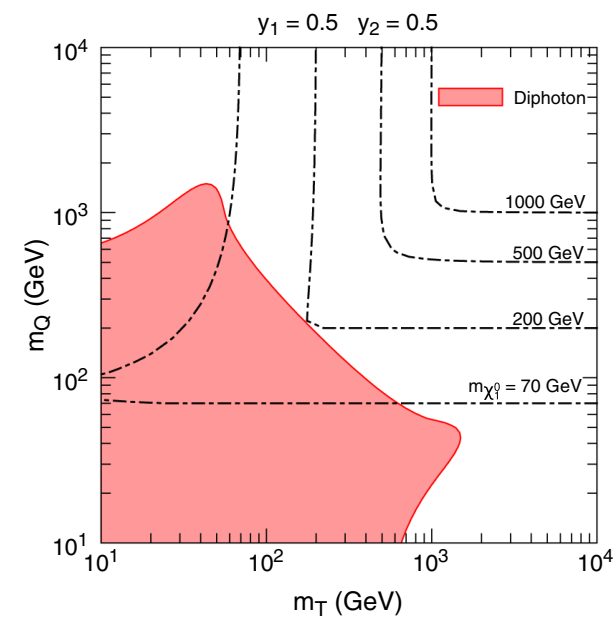

(a) $y_{1}=y_{2}=0.5$

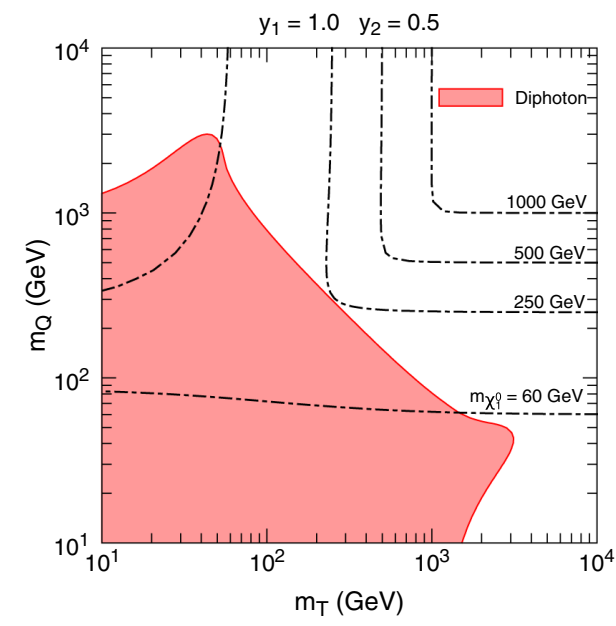

(b) $y_{1}=1.0, y_{2}=0.5$

FIG. 11. Expected 95\% exclusion regions in the $m_{T}-m_{O}$ plane from the CEPC $h \rightarrow \gamma \gamma$ measurement with an integrated luminosity of $3 \mathrm{ab}^{-1}$ for the fixed Yukawa couplings of $y_{1}=y_{2}=0.5$ (a) and $\left(y_{1}, y_{2}\right)=(1.0,0.5)$ (b). Dot-dashed lines denote contours of $m_{\chi_{1}^{0}}$.

$$
\begin{aligned}
A_{1 / 2}(\tau) & =2[\tau+(\tau-1) f(\tau)] \tau^{-2}, \\
A_{1}(\tau) & =-\left[2 \tau^{2}+3 \tau+3(2 \tau-1) f(\tau)\right] \tau^{-2},
\end{aligned}
$$

where the function $f(\tau)$ and the parameters $\tau$ are defined as

$$
\begin{gathered}
f(\tau)= \begin{cases}\arcsin ^{2} \sqrt{\tau}, & \tau \leq 1, \\
-\frac{1}{4}\left[\log \frac{1+\sqrt{1-\tau^{-1}}}{1-\sqrt{1-\tau^{-1}}}-i \pi\right]^{2}, & \tau>1,\end{cases} \\
\tau_{W} \equiv \frac{m_{h}^{2}}{4 m_{W}^{2}}, \quad \tau_{f} \equiv \frac{m_{h}^{2}}{4 m_{f}^{2}}, \quad \tau_{\chi_{i}^{ \pm}} \equiv \frac{m_{h}^{2}}{4 m_{\chi_{i}^{ \pm}}^{2}} .
\end{gathered}
$$

Using the above formula, we calculate the prediction for $\kappa_{\gamma}$ in the TQDM model and compare it with the CEPC precision. In Fig. 11, the red regions are expected to be excluded by the CEPC $h \rightarrow \gamma \gamma$ measurement at 95\% C.L. We can see that it is possible to explore up to $\chi_{1}^{0} \sim$ 200-250 GeV through this measurement. Compared with the results for the $e^{+} e^{-} \rightarrow Z h$ measurement shown in Figs. 9(a) and 9(b), we find that the $h \rightarrow \gamma \gamma$ measurement has less capability to investigate the parameter regions with $m_{Q} \gg m_{T}$ or $m_{T} \gg m_{Q}$.

\section{CONCLUSIONS AND DISCUSSIONS}

In this paper, we investigate the current constraints on the TQDM model from $13 \mathrm{TeV}$ LHC searches, and further 
study the prospects of searches at future colliders, including the SPPC and the CEPC. As the dark sector fermions could be directly produced at high energy hadron colliders, we discuss three signal channels, i.e., the monojet $+E_{\mathrm{T}}$, disappearing track, and multilepton $+E_{\mathrm{T}}$ channels, at the LHC and the SPPC.

In the monojet $+E_{\mathrm{T}}$ channel, we find that when $\chi_{1}^{0}$ is almost pure triplet (quadruplet), the LHC search has excluded the parameter regions with $m_{\chi_{1}^{0}} \lesssim 70(200) \mathrm{GeV}$ at $95 \%$ C.L. Because of the extremely high collision energy, the monojet $+\mathscr{E}_{\mathrm{T}}$ search at the SPPC will be able to explore most of the parameter regions allowed by the observed DM relic density, up to $m_{\chi_{1}^{0}} \sim 1000-2000 \mathrm{GeV}$.

If $\chi_{1}^{ \pm}$is nearly degenerate in mass with $\chi_{1}^{0}$, it would have a moderate lifetime that leads to the disappearing track signal at colliders. This case can be realized in the regions with $\left|y_{1}\right|=\left|y_{2}\right|$, or $m_{T} \gg \max \left(m_{Q},\left|y_{1}\right| v,\left|y_{2}\right| v\right)$, or $m_{Q} \gg \max \left(m_{T},\left|y_{1}\right| v,\left|y_{2}\right| v\right)$. We find that for a pure triplet (quadruplet) $\chi_{1}^{0}$, the parameter regions with $m_{\chi_{1}^{0}} \lesssim$ 410 (472) $\mathrm{GeV}$ are excluded at 95\% C.L. by the disappearing track search at the LHC, while the SPPC would explore up to $m_{\chi_{1}^{0}} \sim 3200-4500(3500-5200) \mathrm{GeV}$.

The multilepton $+\mathscr{E}_{\mathrm{T}}$ channel is suitable to investigate the parameter regions where the mass spectrum is not compressed. We find that some regions with $m_{\chi_{1}^{0}} \lesssim$ $200 \mathrm{GeV}$ have been excluded by the LHC multilepton + $E_{\mathrm{T}}$ searches, while the same kind of searches at the SPPC will probe the parameter regions up to $m_{\chi_{1}^{0}} \sim 2000 \mathrm{GeV}$. If the mass spectrum is compressed, the discovery capability of this channel is much weaker than that of the monojet + $E_{\mathrm{T}}$ channel.

On the other hand, the future Higgs factory CEPC will be able to study loop effects of BSM physics through high precision Higgs measurements. We calculate the loop correction to the $e^{+} e^{-} \rightarrow Z h$ production cross section induced by dark sector fermions in the TQDM model, and find that the related CEPC measurement would explore up to $m_{\chi_{1}^{0}} \sim 300 \mathrm{GeV}$ for moderate values of $y_{1}$ and $y_{2}$. We also compute the deviation of the $h \rightarrow \gamma \gamma$ partial width induced by $\chi_{1,2,3}^{ \pm}$. The sensitivity of the $h \rightarrow \gamma \gamma$ measurement will be weaker than that of the $e^{+} e^{-} \rightarrow Z h$ measurement, but it covers some particular regions where the latter loses sensitivity due to the threshold effect.

Although we have treated the Yukawa couplings $y_{1}$ and $y_{2}$ as free parameters in the above calculations, large $y_{1}$ and $y_{2}$ may cause a problem. As the Yukawa couplings give negative contributions to the $\beta$ function of the Higgs quartic coupling $\lambda$, sufficient large $y_{1}$ and $y_{2}$ may render $\lambda$ negative at a scale much lower than the Planck scale, endangering the stability of the EW vacuum. In order to evaluate such an effect, we derive the dark sector contributions to the $\beta$ functions of $\lambda, g, g^{\prime}$, and $y_{t}$, the top Yukawa coupling, at one-loop level as

$$
\begin{gathered}
\Delta \beta_{\lambda}=\frac{1}{16 \pi^{2}}\left[8 \lambda\left(y_{1}^{2}+y_{2}^{2}\right)-\frac{28}{9}\left(y_{1}^{4}+y_{2}^{4}\right)-\frac{40}{9} y_{1}^{2} y_{2}^{2}\right], \\
\Delta \beta_{g}=\frac{1}{16 \pi^{2}} 8 g^{3}, \quad \Delta \beta_{g^{\prime}}=\frac{1}{16 \pi^{2}} \frac{4}{3} g^{\prime 3}, \\
\Delta \beta_{y_{t}}=\frac{1}{16 \pi^{2}} 2 y_{t}\left(y_{1}^{2}+y_{2}^{2}\right),
\end{gathered}
$$

while the $\beta$ functions of $y_{1}$ and $y_{2}$ are

$$
\beta_{y_{1}}=\frac{1}{16 \pi^{2}} y_{1}\left(\frac{19}{6} y_{1}^{2}+\frac{10}{3} y_{2}^{2}-\frac{69}{4} g^{2}-\frac{3}{4} g^{\prime 2}+3 y_{t}^{2}\right),
$$

$\beta_{y_{2}}=\frac{1}{16 \pi^{2}} y_{2}\left(\frac{19}{6} y_{2}^{2}+\frac{10}{3} y_{1}^{2}-\frac{69}{4} g^{2}-\frac{3}{4} g^{\prime 2}+3 y_{t}^{2}\right)$.

These expressions are derived by hand and crosschecked using PyR@TE 2.0.0 [91].

By solving the renormalization group equations with the initial values of the couplings at the top mass pole [92], we obtain the running values of the couplings at high scales. According to our calculation, if $\sqrt{y_{1}^{2}+y_{2}^{2}} \lesssim 0.5$, the EW vacuum would be stable up to the Planck scale. If $0.5 \lesssim \sqrt{y_{1}^{2}+y_{2}^{2}} \lesssim 0.7$, the vacuum would be metastable. For an even larger $\sqrt{y_{1}^{2}+y_{2}^{2}}$, some additional bosonic degrees of freedom would be needed above the TeV scale for ensuring the vacuum stability. Surprisingly, introducing new Yukawa couplings in the TQDM model do not make the vacuum stability worse than the SM. The reason is that the large, positive contribution to $\beta_{g}$ from the triplet and quadruplets increase $g$ at high scales, and hence indirectly lift up $\lambda$. Nevertheless, $g$ would reach a Landau pole around the Planck scale. Thus, one may expect that there is other new physics below the Planck scale.

\section{ACKNOWLEDGMENTS}

This work is supported by the National Natural Science Foundation of China under Grants No. 11475189 and No. 11475191, and by the National Key Program for Research and Development (No. 2016YFA0400200). Z.-H. Y. is supported by the Australian Research Council. 
[1] G. Aad et al. (ATLAS Collaboration), Observation of a new particle in the search for the Standard Model Higgs boson with the ATLAS detector at the LHC, Phys. Lett. B 716, 1 (2012).

[2] S. Chatrchyan et al. (CMS Collaboration), Observation of a new boson at a mass of $125 \mathrm{GeV}$ with the CMS experiment at the LHC, Phys. Lett. B 716, 30 (2012).

[3] G. Bertone, D. Hooper, and J. Silk, Particle dark matter: Evidence, candidates and constraints, Phys. Rep. 405, 279 (2005).

[4] J. L. Feng, Dark matter candidates from particle physics and methods of detection, Annu. Rev. Astron. Astrophys. 48, 495 (2010).

[5] B.-L. Young, A survey of dark matter and related topics in cosmology, Front. Phys. (Beijing) 12, 121201 (2017); A survey of dark matter and related topics in cosmology, Front. Phys. (Beijing) 12, 121202(E) (2017).

[6] G. Arcadi, M. Dutra, P. Ghosh, M. Lindner, Y. Mambrini, M. Pierre, S. Profumo, and F. S. Queiroz, The waning of the WIMP? A review of models, searches, and constraints, arXiv:1703.07364.

[7] G. Jungman, M. Kamionkowski, and K. Griest, Supersymmetric dark matter, Phys. Rep. 267, 195 (1996).

[8] M. Cirelli, N. Fornengo, and A. Strumia, Minimal dark matter, Nucl. Phys. B753, 178 (2006).

[9] M. Cirelli and A. Strumia, Minimal dark matter: Model and results, New J. Phys. 11, 105005 (2009).

[10] T. Hambye, F. S. Ling, L. L. Honorez, and J. Rocher, Scalar multiplet dark matter, J. High Energy Phys. 07 (2009) 090; Scalar multiplet dark matter, J. High Energy Phys. 05 (2010) 066.

[11] Y. Cai, W. Chao, and S. Yang, Scalar septuplet dark matter and enhanced $h \rightarrow \gamma \gamma$ decay rate, J. High Energy Phys. 12 (2012) 043.

[12] B. Ostdiek, Constraining the minimal dark matter fiveplet with LHC searches, Phys. Rev. D 92, 055008 (2015).

[13] C. Cai, Z.-M. Huang, Z. Kang, Z.-H. Yu, and H.-H. Zhang, Perturbativity limits for scalar minimal dark matter with Yukawa interactions: Septuplet, Phys. Rev. D 92, 115004 (2015).

[14] E. D. Nobile, M. Nardecchia, and P. Panci, Millicharge or decay: A critical take on minimal dark Matter, J. Cosmol. Astropart. Phys. 04 (2016) 048.

[15] R. Mahbubani and L. Senatore, The Minimal model for dark matter and unification, Phys. Rev. D 73, 043510 (2006).

[16] F. D'Eramo, Dark matter and Higgs boson physics, Phys. Rev. D 76, 083522 (2007).

[17] R. Enberg, P. J. Fox, L. J. Hall, A. Y. Papaioannou, and M. Papucci, LHC and dark matter signals of improved naturalness, J. High Energy Phys. 11 (2007) 014.

[18] T. Cohen, J. Kearney, A. Pierce, and D. Tucker-Smith, Singlet-doublet dark matter, Phys. Rev. D 85, 075003 (2012).

[19] O. Fischer and J. J. van der Bij, The scalar singlet-triplet dark matter model, J. Cosmol. Astropart. Phys. 01 (2014) 032.

[20] C. Cheung and D. Sanford, Simplified models of mixed dark matter, J. Cosmol. Astropart. Phys. 02 (2014) 011.

[21] A. Dedes and D. Karamitros, Doublet-triplet fermionic dark matter, Phys. Rev. D 89, 115002 (2014).
[22] M. A. Fedderke, T. Lin, and L.-T. Wang, Probing the fermionic Higgs portal at lepton colliders, J. High Energy Phys. 04 (2016) 160.

[23] L. Calibbi, A. Mariotti, and P. Tziveloglou, Singlet-doublet model: Dark matter searches and LHC constraints, J. High Energy Phys. 10 (2015) 116.

[24] A. Freitas, S. Westhoff, and J. Zupan, Integrating in the Higgs portal to fermion dark matter, J. High Energy Phys. 09 (2015) 015.

[25] C. E. Yaguna, Singlet-doublet dirac dark matter, Phys. Rev. D 92, 115002 (2015).

[26] T. M. P. Tait and Z.-H. Yu, Triplet-quadruplet dark matter, J. High Energy Phys. 03 (2016) 204.

[27] S. Horiuchi, O. Macias, D. Restrepo, A. Rivera, O. Zapata, and H. Silverwood, The Fermi-LAT gamma-ray excess at the Galactic Center in the singlet-doublet fermion dark matter model, J. Cosmol. Astropart. Phys. 03 (2016) 048.

[28] S. Banerjee, S. Matsumoto, K. Mukaida, and Y.-L. S. Tsai, WIMP dark matter in a well-tempered Regime: A case study on singlet-doublets fermionic WIMP, J. High Energy Phys. 11 (2016) 070.

[29] C. Cai, Z.-H. Yu, and H.-H. Zhang, CEPC precision of electroweak oblique parameters and weakly interacting dark matter: The fermionic case, Nucl. Phys. B921, 181 (2017).

[30] T. Abe, Effect of $C P$ violation in the singlet-doublet dark matter model, Phys. Lett. B 771, 125 (2017).

[31] W.-B. Lu and P.-H. Gu, Mixed inert scalar triplet dark matter, radiative neutrino masses and leptogenesis, Nucl. Phys. B924, 279 (2017).

[32] C. Cai, Z.-H. Yu, and H.-H. Zhang, CEPC precision of electroweak oblique parameters and weakly interacting dark matter: The scalar case, Nucl. Phys. B924, 128 (2017).

[33] N. Maru, T. Miyaji, N. Okada, and S. Okada, Fermion dark matter in gauge-Higgs unification, J. High Energy Phys. 07 (2017) 048.

[34] X. Liu and L. Bian, Dark matter and electroweak phase transition in the mixed scalar dark matter model, arXiv: 1706.06042 .

[35] D. Egana-Ugrinovic, The minimal fermionic model of electroweak baryogenesis, J. High Energy Phys. 12 (2017) 064.

[36] Q.-F. Xiang, X.-J. Bi, P.-F. Yin, and Z.-H. Yu, Exploring fermionic dark matter via Higgs precision measurements at the circular electron positron collider, arXiv:1707.03094.

[37] A. Voigt and S. Westhoff, Virtual signatures of dark sectors in Higgs couplings, J. High Energy Phys. 11 (2017) 009.

[38] CEPC-SPPC Study Group Collaboration, Reports No. IHEP-CEPC-DR-2015-01, No. IHEP-TH-2015-01, No. IHEP-EP-2015-01.

[39] CEPC-SPPC Study Group Collaboration, Reports No. IHEP-CEPC-DR-2015-01, No. IHEP-AC-2015-01.

[40] M. Mangano, Physics at the FCC-hh, a $100 \mathrm{TeV}$ pp collider, arXiv: 1710.06353 .

[41] M. Beltran, D. Hooper, E. W. Kolb, Z. A. C. Krusberg, and T. M. P. Tait, Maverick dark matter at colliders, J. High Energy Phys. 09 (2010) 037.

[42] P. J. Fox, R. Harnik, J. Kopp, and Y. Tsai, Missing energy signatures of dark matter at the LHC, Phys. Rev. D 85, 056011 (2012). 
[43] M. Aaboud et al. (ATLAS Collaboration), Search for new phenomena in final states with an energetic jet and large missing transverse momentum in $p p$ collisions at $\sqrt{s}=$ $13 \mathrm{TeV}$ using the ATLAS detector, Phys. Rev. D 94, 032005 (2016).

[44] ATLAS Collaboration, Report No. ATLAS-CONF-2017060.

[45] M. Low and L.-T. Wang, Neutralino dark matter at $14 \mathrm{TeV}$ and $100 \mathrm{TeV}$, J. High Energy Phys. 08 (2014) 161.

[46] ATLAS Collaboration, Report No. ATLAS-CONF-2017017.

[47] G. Aad et al. (ATLAS Collaboration), Search for charginos nearly mass degenerate with the lightest neutralino based on a disappearing-track signature in pp collisions at $\sqrt{\mathrm{s}}=$ $8 \mathrm{TeV}$ with the ATLAS detector, Phys. Rev. D 88, 112006 (2013).

[48] M. Cirelli, F. Sala, and M. Taoso, Wino-like Minimal Dark Matter and future colliders, J. High Energy Phys. 10 (2014) 033; Wino-like Minimal Dark Matter and future colliders, J. High Energy Phys. 01 (2015) 041.

[49] H. Fukuda, N. Nagata, H. Otono, and S. Shirai, Higgsino dark matter or not: Role of disappearing track searches at the LHC and future colliders, arXiv:1703.09675.

[50] R. Mahbubani, P. Schwaller, and J. Zurita, Closing the window for compressed dark sectors with disappearing charged tracks, J. High Energy Phys. 06 (2017) 119; Closing the window for compressed dark sectors with disappearing charged tracks, J. High Energy Phys. 10 (2017) 061.

[51] ATLAS Collaboration, Report No. ATLAS-CONF-2017039.

[52] CMS Collaboration, Report No. CMS-PAS-SUS-16-039.

[53] H. Baer, T. Barklow, K. Fujii, Y. Gao, A. Hoang, S. Kanemura, J. List, H. E. Logan, A. Nomerotski, M. Perelstein et al., The International Linear Collider Technical Design Report, Volume 2: Physics, arXiv:1306.6352.

[54] M. Bicer et al. (TLEP Design Study Working Group Collaboration), First look at the physics case of TLEP, J. High Energy Phys. 01 (2014) 164.

[55] M. McCullough, Indirect model-dependent probe of the Higgs self-coupling, Phys. Rev. D 90, 015001 (2014); Indirect model-dependent probe of the Higgs self-coupling, Phys. Rev. D 92, 039903(E) (2015).

[56] C. Shen and S.-h. Zhu, Anomalous Higgs-top coupling pollution of the triple Higgs coupling extraction at a future high-luminosity electron-positron collider, Phys. Rev. D 92, 094001 (2015).

[57] N. Baro and F. Boudjema, Automatized full one-loop renormalization of the MSSM II: The chargino-neutralino sector, the sfermion sector and some applications, Phys. Rev. D 80, 076010 (2009).

[58] A. Denner, Techniques for calculation of electroweak radiative corrections at the one loop level and results for W physics at LEP-200, Fortsch. Phys. 41, 307 (1993).

[59] T. Fritzsche and W. Hollik, Complete one loop corrections to the mass spectrum of charginos and neutralinos in the MSSM, Eur. Phys. J. C 24, 619 (2002).

[60] A. Alloul, N. D. Christensen, C. Degrande, C. Duhr, and B. Fuks, FeynRules 2.0: A complete toolbox for treelevel phenomenology, Comput. Phys. Commun. 185, 2250 (2014).
[61] J. Alwall, R. Frederix, S. Frixione, V. Hirschi, F. Maltoni, O. Mattelaer, H. S. Shao, T. Stelzer, P. Torrielli, and M. Zaro, The automated computation of tree-level and next-toleading order differential cross sections, and their matching to parton shower simulations, J. High Energy Phys. 07 (2014) 079.

[62] T. Sjostrand, S. Mrenna, and P.Z. Skands, PYTHIA 6.4 physics and manual, J. High Energy Phys. 05 (2006) 026.

[63] M. L. Mangano, M. Moretti, F. Piccinini, and M. Treccani, Matching matrix elements and shower evolution for topquark production in hadronic collisions, J. High Energy Phys. 01 (2007) 013.

[64] J. de Favereau, C. Delaere, P. Demin, A. Giammanco, V. Lemaître, A. Mertens, and M. Selvaggi (DELPHES 3 Collaboration), DELPHES 3, A modular framework for fast simulation of a generic collider experiment, J. High Energy Phys. 02 (2014) 057.

[65] N. Arkani-Hamed, S. Dimopoulos, and G. R. Dvali, The hierarchy problem and new dimensions at a millimeter, Phys. Lett. B 429, 263 (1998).

[66] Z.-H. Yu, X.-J. Bi, Q.-S. Yan, and P.-F. Yin, Detecting light stop pairs in coannihilation scenarios at the LHC, Phys. Rev. D 87, 055007 (2013).

[67] Q.-F. Xiang, X.-J. Bi, P.-F. Yin, and Z.-H. Yu, Searches for dark matter signals in simplified models at future hadron colliders, Phys. Rev. D 91, 095020 (2015).

[68] M. Backovic, K. Kong, and M. McCaskey, MadDM v.1.0: Computation of Dark Matter Relic Abundance Using MadGraph5, Phys. Dark Universe 5-6, 18 (2014).

[69] P. A. R. Ade et al. (Planck Collaboration), Planck 2015 results. XIII. Cosmological parameters, Astron. Astrophys. 594, A13 (2016).

[70] G. F. Giudice, M. A. Luty, H. Murayama, and R. Rattazzi, Gaugino mass without singlets, J. High Energy Phys. 12 (1998) 027.

[71] L. Randall and R. Sundrum, Out of this world supersymmetry breaking, Nucl. Phys. B557, 79 (1999).

[72] C. H. Chen, M. Drees, and J.F. Gunion, Addendum/ Erratum: Searching for invisible and almost invisible particles at $e^{+} e^{-}$colliders [arXiv:hep-ph/9512230] and A nonstandard string/SUSY scenario and its phenomenological implications [arXiv:hep-ph/9607421], arXiv:hep-ph/ 9902309.

[73] G. Aad et al. (ATLAS Collaboration), Search for direct production of charginos and neutralinos in events with three leptons and missing transverse momentum in $\sqrt{s}=8 \mathrm{TeV}$ $p p$ collisions with the ATLAS detector, J. High Energy Phys. 04 (2014) 169.

[74] Q.-F. Xiang, X.-J. Bi, P.-F. Yin, and Z.-H. Yu, Searching for singlino-Higgsino dark matter in the NMSSM, Phys. Rev. D 94, 055031 (2016).

[75] ATLAS Collaboration, Report No. ATLAS-CONF-2016096.

[76] C. G. Lester and D. J. Summers, Measuring masses of semiinvisibly decaying particles pair produced at hadron colliders, Phys. Lett. B 463, 99 (1999).

[77] A. Barr, C. Lester, and P. Stephens, m(T2): The truth behind the glamour, J. Phys. G 29, 2343 (2003).

[78] H.-C. Cheng and Z. Han, Minimal kinematic constraints and m(T2), J. High Energy Phys. 12 (2008) 063. 
[79] J. Fleischer and F. Jegerlehner, Radiative corrections to Higgs production by $e^{+} e^{-} \rightarrow Z H$ in the Weinberg-Salam model, Nucl. Phys. B216, 469 (1983).

[80] B. A. Kniehl, Radiative corrections for associated $\mathrm{ZH}$ production at future $e^{+} e^{-}$colliders, Z. Phys. C 55, 605 (1992).

[81] A. Denner, J. Kublbeck, R. Mertig, and M. Bohm, Electroweak radiative corrections to $e^{+} e^{-} \rightarrow H Z$, Z. Phys. C 56, 261 (1992).

[82] Y. Gong, Z. Li, X. Xu, L. L. Yang, and X. Zhao, Mixed QCD-EW corrections for Higgs boson production at $e^{+} e^{-}$ colliders, Phys. Rev. D 95, 093003 (2017).

[83] Q.-F. Sun, F. Feng, Y. Jia, and W.-L. Sang, Mixed electroweak-QCD corrections to $e^{+} e^{-} \rightarrow H Z$ at Higgs factory, Phys. Rev. D 96, 051301 (2017).

[84] X. Mo, G. Li, M.-Q. Ruan, and X.-C. Lou, Physics cross sections and event generation of $e^{+} e^{-}$annihilations at the CEPC, Chin. Phys. C 40, 033001 (2016).

[85] T. Hahn, Generating Feynman diagrams and amplitudes with FeynArts 3, Comput. Phys. Commun. 140, 418 (2001).
[86] T. Hahn and M. Perez-Victoria, Automatized one loop calculations in four-dimensions and D-dimensions, Comput. Phys. Commun. 118, 153 (1999).

[87] G. J. van Oldenborgh, FF: A Package to evaluate one loop Feynman diagrams, Comput. Phys. Commun. 66, 1 (1991).

[88] J. R. Ellis, M. K. Gaillard, and D. V. Nanopoulos, A phenomenological profile of the Higgs boson, Nucl. Phys. B106, 292 (1976).

[89] M. A. Shifman, A. I. Vainshtein, M. B. Voloshin, and V. I. Zakharov, Low-energy theorems for Higgs boson couplings to photons, Yad. Fiz. 30, 1368 (1979) [Sov. J. Nucl. Phys. 30, 711 (1979)].

[90] A. Djouadi, The Anatomy of electroweak symmetry breaking. I: The Higgs boson in the standard model, Phys. Rep. 457, 1 (2008).

[91] F. Lyonnet and I. Schienbein, PyR@TE 2: A Python tool for computing RGEs at two-loop, Comput. Phys. Commun. 213, 181 (2017).

[92] D. Buttazzo, G. Degrassi, P. P. Giardino, G. F. Giudice, F. Sala, A. Salvio, and A. Strumia, Investigating the near-criticality of the Higgs boson, J. High Energy Phys. 12 (2013) 089. 